Pure and Applied Mathematics Quarterly

Volume 7, Number 3

(Special Issue: In honor of

Jacques Tits)

$745-782,2011$

\title{
Thin Severi-Brauer Varieties
}

\author{
Max-Albert Knus and Jean-Pierre Tignol \\ À Jacques Tits, pour son $80^{\text {ème }}$ anniversaire
}

\begin{abstract}
Severi-Brauer varieties are twisted forms of projective spaces (in the sense of Galois cohomology) and are associated in a functorial way to central simple algebras. Similarly quadrics are related to algebras with involution. Since thin projective spaces are finite sets, thin Severi-Brauer varieties are finite sets endowed with a Galois action; they are associated to étale algebras. Similarly, thin quadrics are étale algebras with involution. We discuss embeddings of thin Severi-Brauer varieties and thin quadrics in Severi-Brauer varieties and quadrics as geometric analogues of embeddings of étale algebras into central simple algebras (with or without involution), and consider the geometric counterpart of the Clifford algebra construction. Keywords: Central simple algebra with involution, étale algebra, Clifford algebra, Severi-Brauer variety, thin projective space, thin quadric, field of characteristic one, Galois cohomology
\end{abstract}

\section{INTRODUCTION}

Paraphrasing Tits [18], "thin" geometric objects are characterised by the fact that their automorphism groups are the Weyl groups of the groups of automorphisms of the corresponding classical geometric objects. ${ }^{*}$ Thus a thin $(n-1)$-dimensional

Received November 4, 2009.

The second author is supported in part by the F.R.S.-FNRS (Belgium)

*In [16] Tits says that such objects are defined over the "field of characteristic 1". 
projective space is a finite set of $n$ elements and a $2(n-1)$-dimensional thin quadric is a double covering of a set of $n$ elements.

Our starting point is to consider twisted forms (in the sense of Galois cohomology) of projective spaces and quadrics. Fixing a base field, a twist is given by a continuous action of the absolute Galois group of the field. Twisted forms of projective spaces over a field $F$ occur as Severi-Brauer varieties associated to central simple algebras over $F$, and twisted quadrics correspond to central simple algebras with quadratic pair. Similarly, twisted forms of finite sets are associated to algebraic objects, namely étale algebras, by a correspondence known as Grothendieck's version of Galois theory. Double coverings correspond to étale algebras with involution. Moreover the Grassmannian of maximal linear subspaces of a split quadric of dimension $2(n-1)$ corresponds as a geometric object to the Clifford algebra of a central algebra with quadratic pair as algebraic object. We call this Grassmannian the Clifford set of the quadric.

The first aim of the paper is to study equivariant embeddings of finite twisted objects into corresponding classical objects. For example a general embedding of a finite set of $n$ elements in a projective space of dimension $n-1$ corresponds to the embedding of an étale algebra of rank $n$ in a central simple algebra of degree $n$ (or to the embedding of a torus of rank $n-1$ into a twisted form of $\mathrm{SL}_{\mathrm{n}}$, see $\left.[13, \mathrm{~S} 2]\right)$. The second aim is to discuss in detail the geometric side of the Clifford construction, and to relate it to Clifford algebras for étale algebras with involution and for central simple algebras with quadratic pairs.

In Section 2 we consider Severi-Brauer varieties, in Section 3 quadrics, and in Section 4 the various types of Clifford constructions, where we introduce a special type of Severi-Brauer variety to describe the geometry of a Clifford algebra. Finally we sketch in Section 5 a discussion of two exceptional phenomena. First we describe consequences of the fact that the Dynkin diagrams $A_{3}$ and $D_{3}$ are equal. This is related on the geometric side with the Klein quadric and on the algebraic side with the resolution of the equation of degree 4 . Then we look at triality, that is, outer automorphisms of order 3 of $D_{4}$.

One origin of this paper is the observation of Tits [16, p. 287] that the two phenomena described above also occur over the "field of characteristic 1". Also, his short geometric proof of the fact that two quaternion algebras whose tensor 
product is not division share a common quadratic subfield [19] is a beautiful illustration of the power of geometric insights into algebraic questions. Another source of inspiration was [14], where Saltman discusses the embedding of étale algebras with involution into central simple algebras with involution. Pairs consisting of a maximal étale subalgebra of a central simple algebra (and corresponding pairs of algebras with involutions) are also considered in [1] and [13]. Triality in relation with étale algebras is extensively discussed in [9]. We plan to come back to other aspects, in particular to unitary and symplectic analogues, in [10].

\section{SEVEri-Brauer Projective SPACES}

$\Gamma$-projective spaces and central simple algebras. Throughout most of this work, $F$ is an arbitrary field. We denote by $F_{s}$ a separable closure of $F$ and by $\Gamma$ the absolute Galois group $\operatorname{Gal}\left(F_{s} / F\right)$, which is a profinite group.

A $\Gamma$-projective space $P$ of dimension $n-1$ over a field $F$ is a projective space $\mathbb{P}^{n-1}\left(F_{s}\right)$ of dimension $n-1$ endowed with the discrete topology and a continuous action of $\Gamma$ by collineations, denoted by $x \mapsto^{\gamma} x, x \in P, \gamma \in \Gamma$. For every integer $k \in\{1, \ldots, n\}$, the Grassmannian $G_{k-1}(P)$ of $(k-1)$-dimensional linear varieties in $P$ carries an action of $\Gamma$. We view the set:

$$
\bar{P}=\left\{x \in P \mid{ }^{\gamma} x=x \text { for all } \gamma \in \Gamma\right\} \subset P
$$

as a scheme over $F$. The embedding $\bar{P} \subset P$ induces an isomorphism

$$
\beta: \bar{P} \times_{\operatorname{Spec} F} \operatorname{Spec} F_{s} \stackrel{\sim}{\longrightarrow} P
$$

of $\Gamma$-spaces. From now on we shall not distinguish between $P$ and the pair $(\bar{P}, \beta)$. We have a similar construction for Grassmannians.

Continuous actions of $\Gamma$ are related with Galois cohomology, as we now illustrate in the case of $\Gamma$-projective spaces. There is a standard action of $\Gamma$ on $\mathbb{P}^{n-1}\left(F_{s}\right)$ induced by the action of $\Gamma$ on $F_{s}$. We denote it by $x \mapsto \bar{\gamma} x$. This standard action induces in turn a $\Gamma$-action on the group $\mathrm{PGL}_{n}\left(F_{s}\right)$ of automorphisms of the projective space $\mathbb{P}^{n-1}\left(F_{s}\right)$, given by $\varphi \mapsto \bar{\gamma}(\varphi)=\bar{\gamma} \varphi \bar{\gamma}^{-1}, \varphi \in \operatorname{PGL}_{n}\left(F_{s}\right)$.

A 1-cocycle of $\Gamma$ with values in $\mathrm{PGL}_{n}$ is a continuous map $\Gamma \rightarrow \operatorname{PGL}_{n}\left(F_{s}\right)$, $\gamma \mapsto a_{\gamma}$, such that

$$
a_{\gamma \delta}=a_{\gamma} \bar{\gamma}\left(a_{\delta}\right) \quad \text { for all } \gamma, \delta \in \Gamma
$$


Two cocycles $a_{\gamma}, a_{\gamma}^{\prime}$ are cohomologous or equivalent if there exists $a \in \operatorname{PGL}_{n}\left(F_{s}\right)$ such that $a_{\gamma}^{\prime}=a a_{\gamma} \bar{\gamma}(a)^{-1}$. We denote by $H^{1}\left(\Gamma, \mathrm{PGL}_{n}\right)$ the set of equivalence classes of 1-cocycles.

Let $P=(\bar{P}, \beta)$ be any $\Gamma$-projective space. The map $a_{\gamma}=\beta \gamma \beta^{-1} \bar{\gamma}^{-1}, \gamma \in \Gamma$, is an element of $\operatorname{PGL}_{n}\left(F_{s}\right)$ and defines a 1-cocycle. Moreover one shows that isomorphic $\Gamma$-projective spaces lead to cohomologous cocycles. Let $\operatorname{Proj}_{\Gamma}^{n-1}$ be the groupoid $^{\dagger}$ of $\Gamma$-projective spaces and let Iso $\left(\operatorname{Proj}_{\Gamma}^{n-1}\right)$ be its set of isomorphism classes.

Proposition 2.1. The map $(P, \beta) \mapsto a_{\gamma}$ induces a bijection

$$
\text { Iso }\left(\operatorname{Proj}_{\Gamma}^{n-1}\right) \stackrel{\sim}{\longrightarrow} H^{1}\left(\Gamma, \mathrm{PGL}_{n}\right)
$$

Proof. We describe a map in the opposite direction and refer to [15, p. 161] for details. Given a 1-cocycle $\left(a_{\gamma}\right)_{\gamma \in \Gamma}$, we define a $\Gamma$-action on $P=\mathbb{P}^{n-1}\left(F_{s}\right)$ as

$$
x \mapsto^{\gamma} x=^{a_{\gamma} \bar{\gamma}} x, \quad x \in P .
$$

The $\Gamma$-projective spaces we consider arise from the following construction. Recall that an $F$-algebra $A$ is central simple of degree $n$ if there exists an isomorphism $\alpha: A \otimes_{F} F_{s} \stackrel{\sim}{\longrightarrow} \operatorname{End}_{F_{s}}(V)$ for a vector space $V$ of dimension $n$ over $F_{s}$. Since the group of $F_{s}$-automorphisms of $\operatorname{End}_{F_{s}}(V)$ is isomorphic to $\operatorname{PGL}_{n}\left(F_{s}\right)$, a 1cocycle defing a $\Gamma$-projective space of dimension $n-1$ is also a 1-cocycle defining a central simple algebra of degree $n$. Thus denoting by $\operatorname{CSA}_{F}^{n}$ the groupoid of central simple algebras of degree $n$, the sets Iso $\left(\operatorname{Proj}_{\Gamma}^{n-1}\right)$ and Iso $\left(\operatorname{CSA}_{F}^{n}\right)$ are in bijection, since they are in bijection with $H^{1}\left(\Gamma, \mathrm{PGL}_{n}\right)$.

There is a canonical way to associate a $\Gamma$-projective space to a central simple algebra. Let $\alpha: A \otimes_{F} F_{s} \stackrel{\sim}{\longrightarrow} \operatorname{End}_{F_{s}}(V)$ as above. Since the $n$-dimensional right ideals of $\operatorname{End}_{F_{s}}(V)$ are of the form $\operatorname{Hom}_{F_{s}}(V, U)$ for $U \subset V$ a 1-dimensional subspace, any isomorphism $A_{s} \simeq \operatorname{End}_{F_{s}}(V)$ defines a one-to-one correspondence between $n$-dimensional right ideals of $A_{s}$ and 1-dimensional subspaces of $V$. Therefore, we may canonically associate to $A$ a projective space $\mathcal{S B}(A)$ over $F_{s}$, whose $(k-1)$ dimensional linear varieties are the $n k$-dimensional right ideals of $A_{s}$. The action of the group $\Gamma$ of $F_{s}$ over $F$ induces a continuous action on $A_{s}$, hence also on the right ideals of $A_{s}$, and endows $\mathcal{S B}(A)$ with a $\Gamma$-projective space structure. We

\footnotetext{
${ }^{\dagger} \mathrm{A}$ groupoid is a category in which all morphisms are isomorphisms.
} 
call $\mathcal{S B}(A)$ (or its associated scheme $\overline{\mathcal{S B}(A)}$ over $F$ ) the Severi-Brauer variety of $A$. Its dimension is $n-1$, where $n=\operatorname{deg} A$.

Proposition 2.2. The rule $A \mapsto \mathcal{S B}(A)$ induces an anti-equivalence

$$
\mathrm{CSA}_{F}^{n} \equiv \operatorname{Proj}_{\Gamma}^{n-1}
$$

Proof. Applying [11, Theorem 1, p. 93] it suffices to show that the functor $\mathcal{S B}$ is fully faithfull and essentially surjective. We refer to [6] for details.

$\Gamma$-sets and étale algebras. Finite sets with a continuous left action of $\Gamma$ (for the discrete topology) are called (finite) $\Gamma$-sets. They form a category $\operatorname{Set}_{\Gamma}$ whose morphisms are the $\Gamma$-equivariant maps. Finite direct products and direct sums (= disjoint unions) are defined in this category. We denote by $|X|$ the cardinality of any finite set $X$.

Following Tits [18] we define a thin $\Gamma$-projective space of dimension $n-1$ as a $\Gamma$-set $X$ of $n$ elements. A thin $(k-1)$-dimensional linear subvariety is a $\Gamma$-subset of $X$ with $k$ elements. For every integer $k, 1 \leq k \leq n$, the $\Gamma$-set

$$
\begin{aligned}
G r_{k-1}(X) & =\{(k-1) \text {-dimensional linear varieties contained in } X\} \\
& =\{k \text {-element subsets of } X\}
\end{aligned}
$$

is a thin $\Gamma$-Grassmannian. As for $\Gamma$-projective spaces over $F$, we shall not distinguish between a finite $\Gamma$-set $X$ and its scheme $\bar{X}$ over $F$ of fixed points of the $\Gamma$-action. Like $\Gamma$-projective spaces over $F$, thin $\Gamma$-projective spaces are related with algebraic structures. We recall that a finite-dimensional commutative $F$-algebra $L$ is called étale (over $F$ ) if $L \otimes_{F} F_{s}$ is isomorphic to the $F_{s}$-algebra $F_{s}^{n}=F_{s} \times \cdots \times F_{s}(n$ factors $)$ for some $n \geq 1$. Étale $F$-algebras are the direct products of finite separable field extensions of $F$. We refer to $[7, \S 18$.A $]$ for various equivalent characterizations of étale $F$-algebras. These algebras (with $F$-algebra homomorphisms) form a category Ét $_{F}$ in which finite direct products and finite direct sums (= tensor products) are defined.

For any étale $F$-algebra $L$ of dimension $n$, the set of $F$-algebra homomorphisms

$$
\mathbf{X}(L)=\operatorname{Hom}_{F-\text { alg }}\left(L, F_{s}\right)
$$


is a $\Gamma$-set of $n$ elements since $\Gamma$ acts on $F_{s}$. Conversely, if $X$ is a $\Gamma$-set of $n$ elements, the $F$-algebra $\mathbf{M}(X)$ of $\Gamma$-equivariant maps $X \rightarrow F_{s}$ is an étale $F$ algebra of dimension $n$,

$$
\mathbf{M}(X)=\left\{f: X \rightarrow F_{s} \mid \gamma(f(x))=f\left({ }^{\gamma} x\right) \text { for } \gamma \in \Gamma, x \in X\right\} .
$$

As first observed by Grothendieck, there are canonical isomorphisms

$$
\mathbf{M}(\mathbf{X}(L)) \cong L, \quad \mathbf{X}(\mathbf{M}(X)) \cong X,
$$

so that the functors $\mathbf{M}$ and $\mathbf{X}$ define an anti-equivalence of categories ${ }^{\ddagger}$

$$
\operatorname{Set}_{\Gamma} \equiv \mathrm{Ét}_{F}
$$

(see [2, Prop. (4.3), p. 25] or [7, (18.4)]), which we refer to as the Grothendieck correspondence. Under this anti-equivalence, the cardinality of $\Gamma$-sets corresponds to the dimension of étale $F$-algebras. The disjoint union $\sqcup$ in $\operatorname{Set}_{\Gamma}$ corresponds to the product $\times$ in Ét $t_{F}$ and the product $\times$ in $\operatorname{Set}_{\Gamma}$ to the tensor product $\otimes$ in $\mathrm{Ét}_{F}$. For any integer $n \geq 1$, we let $\mathrm{Et}_{F}^{n}$ denote the groupoid whose objects are $n$ dimensional étale $F$-algebras and whose morphisms are $F$-algebra isomorphisms, and $\operatorname{Set}_{\Gamma}^{n}$ the groupoid of $\Gamma$-sets with $n$ elements. The anti-equivalence (2.3) restricts to an anti-equivalence $\operatorname{Set}_{\Gamma}^{n} \equiv \mathrm{Et}_{F}^{n}$. Isomorphism classes are in bijection with $H^{1}\left(\Gamma, \mathfrak{S}_{n}\right)$, where $\Gamma$ acts trivially on $\mathfrak{S}_{n}$ (see $[7,29.9]$ ).

The split étale algebra $F^{n}$ corresponds to the $\Gamma$-set $\boldsymbol{n}$ of $n$ elements with trivial $\Gamma$-action. Étale algebras of dimension 2 are also called quadratic étale algebras.

Embeddings of $\Gamma$-sets in Severi-Brauer varieties. For $X$ a $\Gamma$-set of $n$ elements and a $\Gamma$-projective space $P$ of dimension $n-1$, we define a general embedding

$$
\varepsilon: X \hookrightarrow P
$$

to be a $\Gamma$-equivariant injective map whose image consists of $n$ points in general position, i.e., not contained in a proper subspace ${ }^{\S}$. The map $\varepsilon$ then induces a map

$$
G r_{k-1} \varepsilon: G r_{k-1}(X) \hookrightarrow G r_{k-1}(P) \quad \text { for } 1 \leq k \leq n,
$$

\footnotetext{
${ }^{\ddagger}$ We let morphisms of $\Gamma$-sets act on the right of the arguments (with the exponential notation) and use the usual function notation for morphisms in the anti-equivalent category of étale algebras.

${ }^{\S}$ Such embeddings are called frames in [18].
} 
which carries each $k$-element subset of $X$ to the linear variety spanned by its image. In the rest of this subsection, we relate $F$-algebra embeddings of $n$ dimensional étale $F$-algebras in a central simple $F$-algebra $A$ with general embeddings of $\Gamma$-sets of $n$ elements in $\mathcal{S B}(A)$. We first discuss the case where the étale $F$-algebra is split.

Proposition 2.4. Let $L$ be a split étale F-algebra of dimension n, and let $A$ be a central simple $F$-algebra of degree $n$. For every F-algebra embedding $\varepsilon: L \hookrightarrow A$, there is an F-algebra isomorphism $\varphi: A \rightarrow \operatorname{End}_{F} L$ such that $\varphi \circ \varepsilon: L \hookrightarrow \operatorname{End}_{F} L$ is the regular representation, which maps each $x \in L$ to multiplication by $x$.

Proof. Let $\left(e_{i}\right)_{1 \leq i \leq n}$ be the collection of primitive idempotents of $L$. We have

$$
A=\varepsilon\left(e_{1}\right) A \oplus \cdots \oplus \varepsilon\left(e_{n}\right) A .
$$

Since the dimension of every right ideal of $A$ is a multiple of $n \operatorname{Ind} A$, we must have

$$
\operatorname{dim} \varepsilon\left(e_{i}\right) A=n \text { for } 1 \leq i \leq n, \quad \text { and } \quad \text { Ind } A=1 .
$$

Identifying $A=\operatorname{End}_{F} V$ for some $F$-vector space $V$ of dimension $n$, we may view $\varepsilon\left(e_{1}\right), \ldots, \varepsilon\left(e_{n}\right)$ as projections on 1-dimensional subspaces $V_{1}, \ldots, V_{n}$ such that $V=V_{1} \oplus \cdots \oplus V_{n}$. Any $F$-vector space isomorphism $V \rightarrow L$ that maps $V_{i}$ to $e_{i} L$ for all $1 \leq i \leq n$ determines an $F$-algebra isomorphism $\varphi: A \rightarrow \operatorname{End}_{F} L$ as required.

Now, let $L$ be an arbitrary $n$-dimensional étale $F$-algebra. Consider an $F$-algebra embedding into a central simple $F$-algebra of degree $n$ :

$$
\varepsilon: L \hookrightarrow A .
$$

For every $\xi \in \mathbf{X}(L):=\operatorname{Hom}_{F \text {-alg }}\left(L, F_{s}\right)$, set

$$
\varepsilon_{*}(\xi)=\left\{x \in A_{s} \mid(1 \otimes \xi(\ell)) \cdot x=(\varepsilon(\ell) \otimes 1) \cdot x \text { for all } \ell \in L\right\} .
$$

Proposition 2.6. The right ideal $\varepsilon_{*}(\xi)$ is n-dimensional, and $\varepsilon_{*}$ is a general embedding

$$
\varepsilon_{*}: \mathbf{X}(L) \hookrightarrow \mathcal{S B}(A)
$$

Proof. Let $L_{s}=L \otimes_{F} F_{s}$, a split étale $F_{s}$-algebra, and $\varepsilon_{s}=\varepsilon \otimes \operatorname{Id}_{F_{s}}: L_{s} \hookrightarrow$ $A_{s}$. By Proposition 2.4, we may identify $A_{s}=\operatorname{End}_{F_{s}} L_{s}$ in such a way that 
$\varepsilon_{s}: L_{s} \hookrightarrow \operatorname{End}_{F_{s}} L_{s}$ is the regular representation. Let $\left(e_{i}\right)_{1 \leq i \leq n}$ be the collection of primitive idempotents of $L_{s}$. Using the canonical correspondence $\operatorname{Hom}_{F \text {-alg }}\left(L, F_{s}\right)=\operatorname{Hom}_{F_{s} \text {-alg }}\left(L_{s}, F_{s}\right)$, we may set

$$
\mathbf{X}(L)=\left\{\xi_{1}, \ldots, \xi_{n}\right\}
$$

where $\xi_{i}: L_{s} \rightarrow F_{s}$ maps $e_{i}$ to 1 and $e_{j}$ to 0 for $j \neq i$. For $f \in \operatorname{End}_{F_{s}} L_{s}$, the condition

$$
\left(1 \otimes \xi_{i}(\ell)\right) \cdot f=(\varepsilon(\ell) \otimes 1) \circ f \quad \text { for all } \ell \in L
$$

is equivalent to

$$
\left(1 \otimes \xi_{i}(\ell)\right) \cdot f=\varepsilon_{s}(\ell) \circ f \quad \text { for all } \ell \in L_{s} .
$$

For $\ell=e_{1}, \ldots, e_{n}$, this condition can be rewritten as

$$
e_{j} \cdot f(x)=0 \quad \text { and } \quad e_{i} \cdot f(x)=f(x) \quad \text { for all } x \in L_{s} .
$$

Therefore,

$$
\varepsilon_{*}\left(\xi_{i}\right)=\operatorname{Hom}_{F_{s}}\left(L_{s}, e_{i} L_{s}\right),
$$

which shows that $\operatorname{dim} \varepsilon_{*}\left(\xi_{i}\right)=n$. Moreover,

$$
\varepsilon_{*}\left(\xi_{1}\right)+\cdots+\varepsilon_{*}\left(\xi_{n}\right)=\operatorname{Hom}_{F_{s}}\left(L_{s}, e_{1} L_{s}+\cdots+e_{n} L_{s}\right)=\operatorname{End}_{F_{s}} L_{s} .
$$

Therefore $\varepsilon_{*}\left(\xi_{1}\right), \ldots, \varepsilon_{*}\left(\xi_{n}\right)$ are in general position in the projective space $\mathcal{S B}(A)$.

To complete the proof, we show $\varepsilon_{*}$ is $\Gamma$-equivariant. For $\gamma \in \Gamma$, we have

$$
\gamma\left(\varepsilon_{*}(\xi)\right)=\left\{x \in A_{s} \mid(1 \otimes \xi(\ell)) \cdot \gamma^{-1}(x)=(\varepsilon(\ell) \otimes 1) \cdot \gamma^{-1}(x) \text { for all } \ell \in L\right\} .
$$

By applying $\gamma$ to each side, we see that the condition

$$
(1 \otimes \xi(\ell)) \cdot \gamma^{-1}(x)=(\varepsilon(\ell) \otimes 1) \cdot \gamma^{-1}(x)
$$

is equivalent to

$$
\left(1 \otimes{ }^{\gamma} \xi(\ell)\right) \cdot x=(\varepsilon(\ell) \otimes 1) \cdot x .
$$

Thus, $\gamma\left(\varepsilon_{*}(\xi)\right)=\varepsilon_{*}\left({ }^{\gamma} \xi\right)$.

Conversely, every general embedding $X \hookrightarrow \mathcal{S B}(A)$ of a $\Gamma$-set $X$ of $n$ elements arises from an $F$-algebra embedding, as we now show.

Given $n$-dimensional right ideals $I_{1}, \ldots, I_{n} \subset A_{s}$ such that $\left\{I_{1}, \ldots, I_{n}\right\}$ is stable under $\Gamma$ and $I_{1}+\cdots+I_{n}=A_{s}$, let

$$
L_{s}=\left\{x \in A_{s} \mid x I_{i} \subset I_{i} \text { for } 1 \leq i \leq n\right\} .
$$


The set $L_{s}$ is an $F_{s}$-subalgebra of $A_{s}$ stable under the action of $\Gamma$. Let $L=L_{s}^{\Gamma}$ be the fixed $F$-subalgebra.

Proposition 2.7. The F-algebra $L$ is étale of dimension $n$ and $\left\{I_{1}, \ldots, I_{n}\right\}$ is the image of the general embedding $\varepsilon_{*}: \mathbf{X}(L) \hookrightarrow \mathcal{S B}(A)$ induced by the inclusion $\varepsilon: L \hookrightarrow A$.

Proof. We may identify $A_{s}=M_{n}\left(F_{s}\right)$ in such a way that for $1 \leq i \leq n$ the ideal $I_{i}$ consists of matrices whose nonzero entries are on the $i$-th row. For $x=\left(x_{i j}\right)_{1 \leq i, j \leq n}$ we have $x I_{j} \subset I_{j}$ if and only if $x_{i j}=0$ for $i \neq j$. Therefore, $L_{s}$ is the algebra of diagonal matrices. Since $L_{s}=L \otimes_{F} F_{s}$, it follows that $L$ is an étale $F$-algebra of dimension $n$. Under the canonical identification $\mathbf{X}(L)=$ $\operatorname{Hom}_{F_{s} \text { alg }}\left(L_{s}, F_{s}\right)$ we have $\mathbf{X}(L)=\left\{\xi_{1}, \ldots, \xi_{n}\right\}$ where $\xi_{i}: L_{s} \rightarrow F_{s}$ yields the $i$-th diagonal entry. Matrix computation shows that $\varepsilon_{*}\left(\xi_{i}\right)=I_{i}$.

Remark 2.8. The definition of the map $\varepsilon_{*}$ is inspired by the correspondence between maximal subfields of a division algebra and closed points on the corresponding Severi-Brauer variety set up by Merkurjev in [12].

\section{Quadrics And Algebras with Involution}

$\Gamma$-quadrics and quadratic pairs. As in the preceding section, let $F$ be an arbitrary field and $\Gamma$ the Galois group of some separable closure $F_{s}$ of $F$. We call $\Gamma$-quadric in a $(2 n-1)$-dimensional $\Gamma$-projective space $P$ over $F$ any quadric in $P$ defined by a hyperbolic quadratic form and stable under the action of $\Gamma$. The $\Gamma$-quadrics we consider in the sequel arise from quadratic pairs on central simple algebras, which we now recall from $[7, \S 5]$.

Let $B$ be an arbitrary $F$-algebra. An involution ${ }^{\top}$ on $B$ is an $F$-linear antiautomorphism of $B$ of order 2. The sets of symmetric, skew-symmetric, symmetrized and alternating elements are the following subspaces of $B$ :

$$
\begin{aligned}
& \operatorname{Sym}(B, \sigma)=\{x \in B \mid \sigma(x)=x\} \\
& \operatorname{Skew}(B, \sigma)=\{x \in B \mid \sigma(x)=-x\} \\
& \operatorname{Symd}(B, \sigma)=\{x+\sigma(x) \mid x \in B\} \\
& \operatorname{Alt}(B, \sigma)=\{x-\sigma(x) \mid x \in B\} .
\end{aligned}
$$

\footnotetext{
"We only consider F-linear involutions, which are sometimes called of the first kind, when semi-linear involutions are allowed.
} 
Now, let $A$ be a central simple $F$-algebra of degree $2 n$. A quadratic pair on $A$ is a pair $(\sigma, f)$ where $\sigma$ is an involution on $A$ and $f: \operatorname{Sym}(A, \sigma) \rightarrow F$ is a linear map, subject to the following conditions:

(1) $\operatorname{dim}_{F} \operatorname{Sym}(A, \sigma)=n(2 n+1)$ and $\operatorname{Trd}_{A}(\operatorname{Skew}(A, \sigma))=\{0\} ;$

(2) $f(x+\sigma(x))=\operatorname{Trd}_{A}(x)$ for all $x \in A$.

If $A=\operatorname{End}_{F} V$, any quadratic pair $(\sigma, f)$ on $A$ is attached to a nonsingular quadratic form $q$ on $V$. The involution $\sigma$ is the involution adjoint to the quadratic form $q$ and we refer to $[7, \S 5 \mathrm{~B}]$ for the definition of $f$.

For a central simple algebra $A$ over a field of characteristic not 2 a quadratic pair $(\sigma, f)$ is determined by the involution $\sigma$, which has to be of orthogonal type (see $[7, \S 5 \mathrm{~B}])$.

Any quadratic pair $(\sigma, f)$ on $A$ extends to a quadratic pair $\left(\sigma_{s}, f_{s}\right)$ on $A_{s}=A \otimes_{F}$ $F_{s}$. Since $A_{s}$ is split, we may identify $A_{s}=\operatorname{End}_{F_{s}}(V)$ for some $2 n$-dimensional $F_{s}$-vector space $V$. Then $\left(\sigma_{s}, f_{s}\right)$ is adjoint to some hyperbolic quadratic form $q$ on $V$, which is uniquely determined up to a scalar factor, see [7, (5.11)]. Recall from $[7,(6.5)]$ that a right ideal $I \subset A_{s}$ is called isotropic for $\left(\sigma_{s}, f_{s}\right)$ when

$$
\sigma_{s}(I) \cdot I=\{0\} \quad \text { and } \quad f_{s}\left(I \cap \operatorname{Sym} \sigma_{s}\right)=\{0\} .
$$

Isotropic ideals have the form $\operatorname{Hom}_{F_{s}}(V, W)$ where $W \subset V$ is a totally isotropic subspace of $V$ for the form $q$, by [7, (6.6)]. Therefore, we may consider the quadric $\mathbf{Q}(\sigma, f)$ in $\mathcal{S B}(A)$ whose points are the $2 n$-dimensional right ideals in $A_{s}$ that are isotropic for $\left(\sigma_{s}, f_{s}\right)$. The quadric is stable under the action of the Galois group $\Gamma$ of $F_{s} / F$ and is given by the equation $q=0$ in $\mathbb{P}(V)$ under the identification $\mathcal{S B}(A)=\mathbb{P}(V)$.

Let QCSA ${ }_{F}^{2 n}$ be the groupoid of central simple algebras over $F$ of degree $2 n$ with quadratic pairs and let Quad ${ }_{\Gamma}^{2(n-1)}$ be the groupoid of $\Gamma$-quadrics of dimension $2(n-1)$ embedded in a $\Gamma$-projective space of dimension $2 n-1$. As in Proposition 2.2, we have:

Proposition 3.1. The rule $(A, \sigma, f) \mapsto \mathbf{Q}(\sigma, f)$ induces an anti-equivalence of categories $\mathrm{QCSA}_{F}^{2 n} \equiv \operatorname{Quad}_{\Gamma}^{2(n-1)}$.

The automorphism group of a quadric of dimension $2(n-1)$ over $F_{s}$ or of a central simple algebra of degree $2 n$ with a quadratic pair over $F_{s}$ is the projective 
orthogonal group $\mathrm{PGO}_{2 n}\left(F_{s}\right)$ thus Galois cohomology leads to bijections of the sets Iso $\left(\mathrm{QCSA}_{F}^{2 n}\right)$ and Iso $\left(\operatorname{Quad}_{\Gamma}^{2(n-1)}\right)$ with $H^{1}\left(\Gamma, \mathrm{PGO}_{2 n}\right)$.

Thin $\Gamma$-quadrics and quadratic étale extensions. A morphism of $\Gamma$-sets $Y_{0} \stackrel{\pi}{\leftarrow} Y$ is called a $\Gamma$-covering if the number of elements in each fiber $y_{0}^{\pi^{-1}} \subset Y$ does not depend on $y_{0} \in Y_{0}$. This number is called the degree of the covering. Of particular importance in the sequel are coverings of degree 2 , which are also called double coverings. Each such covering $Y_{0} \stackrel{\pi}{\leftarrow} Y$ defines a canonical automorphism $Y \stackrel{\sigma}{\leftarrow} Y$ of order 2, which interchanges the elements in each fiber of $\pi$. Clearly, this automorphism has no fixed points. We call involution of a $\Gamma$-set with an even number of elements any automorphism of order 2 without fixed points. If $Y$ is a $\Gamma$-set and $Y \stackrel{\sigma}{\leftarrow} Y$ is an involution, the set of orbits

$$
Y / \sigma=\left\{\left\{y, y^{\sigma}\right\} \mid y \in Y\right\}
$$

is a $\Gamma$-set and the canonical map $(Y / \sigma) \leftarrow Y$ is a double covering. Thus, the notions of double covering and involution of $\Gamma$-sets are equivalent. Paraphrasing Tits $[18,7.3]$ we call a double $\Gamma$-covering $Y_{0} \stackrel{\pi}{\leftarrow} Y$ with $\left|Y_{0}\right|=n$, resp. a $\Gamma$-set with involution $(Y, \sigma)$ with $|Y|=2 n$, a thin $2(n-1)$-dimensional $\Gamma$-quadric. Putting $Y=\left\{a_{1}, b_{1}, a_{2}, b_{2}, \ldots, a_{n}, b_{n}\right\}$ where $a_{i}^{\pi}=b_{i}^{\pi}$ for all $i$, we view $Y_{0}$ as the set of pairs $Y_{0}=\left\{\left\{a_{1}, b_{1}\right\},\left\{a_{2}, b_{2}\right\}, \ldots,\left\{a_{n}, b_{n}\right\}\right\}$. An isotropic linear subvariety of dimension $k-1$ is a $k$-element subset of $Y$ not containing any pair $\left\{a_{i}, b_{i}\right\}$. Thus, every isotropic linear subvariety is contained in a maximal isotropic linear subvariety, which has dimension $n-1$. The maximal isotropic linear subvarieties may be viewed as the sections of the map $\pi$; they form a $\Gamma$-set $C\left(Y / Y_{0}\right)$ (or $C(Y, \sigma))$ of $2^{n}$ elements, called the Clifford $\Gamma$-set of $Y / Y_{0}$, which is studied in more detail in $\S 4$.

Étale extensions are defined so as to correspond to $\Gamma$-coverings under the antiequivalence (2.3):

Proposition 3.2. For a homomorphism $L_{0} \stackrel{\varepsilon}{\rightarrow} L$ of étale F-algebras, the following conditions are equivalent:

(a) $\varepsilon$ endows $L$ with a structure of free $L_{0}$-module of rank $d$;

(b) the morphism of $\Gamma$-sets $\mathbf{X}\left(L_{0}\right) \stackrel{\mathbf{X}(\varepsilon)}{\longleftarrow} \mathbf{X}(L)$ is a covering of degree $d$.

Proof. Let $e_{1}, \ldots, e_{r}$ be the primitive idempotents of $L_{0}$, so that $L_{0} \cong M_{1} \times$ $\cdots \times M_{r}$ with $M_{i}=e_{i} L_{0}$ a finite separable field extension of $F$. Then $\varepsilon\left(e_{1}\right)$, 
$\ldots, \varepsilon\left(e_{r}\right)$ are orthogonal idempotents of $L$ and, letting $L_{i}=\varepsilon\left(e_{i}\right) L$ we have $L \cong L_{1} \times \cdots \times L_{r}$ where each $L_{i}$ is an $M_{i}$-vector space. Condition (a) holds if and only if

$$
\operatorname{dim}_{M_{1}} L_{1}=\cdots=\operatorname{dim}_{M_{r}} L_{r}=d
$$

To prove (a) and (b) are equivalent, we may extend scalars to $F_{s}$ and therefore assume $F=F_{s}=M_{i}$ for all $i$. Then $\left(e_{i}\right)_{i=1}^{r}$ is a base of $L_{0}$, and we may identify $\mathbf{X}\left(L_{0}\right)$ with the dual base $\left(e_{i}^{*}\right)_{i=1}^{r}$. The number of elements of $\mathbf{X}(L)$ in the fiber above $e_{i}^{*}$ is the dimension $\operatorname{dim}_{M_{i}} L_{i}$, so (a) and (b) are equivalent.

When the equivalent conditions of Proposition 3.2 hold, the map $\varepsilon$ is injective; identifying $L_{0}$ to a subalgebra of $L$, we call $L$ an étale extension of $L_{0}$ of degree $d$. Étale extensions of degree 2 are called quadratic étale extensions. They can also be defined by means of automorphisms, as we now show.

Let $\sigma: L \rightarrow L$ be an automorphism of order 2 of an étale $F$-algebra $L$, and let $L_{0}=\operatorname{Sym}(L, \sigma) \subset L$ denote the $F$-subalgebra of fixed elements, which is necessarily étale.

Proposition 3.3. The following conditions are equivalent:

(a) the inclusion $L_{0} \hookrightarrow L$ is a quadratic étale extension of $F$-algebras;

(b) the automorphism $\mathbf{X}(\sigma)$ is an involution on $\mathbf{X}(L)$.

Proof. By the anti-equivalence (2.3) we have

$$
\mathbf{X}\left(L_{0}\right)=\mathbf{X}(L) / \mathbf{X}(\sigma) .
$$

Therefore, (a) is equivalent by Proposition 3.2 to the condition that the canonical map $\mathbf{X}(L) / \mathbf{X}(\sigma) \leftarrow \mathbf{X}(L)$ is a $\Gamma$-covering of degree 2 , hence also to (b).

When the equivalent conditions of Proposition 3.3 hold, the automorphism $\sigma$ is called an involution of the étale $F$-algebra $L$, whose dimension is then necessarily even. Thus, the notions of quadratic étale extension and involution of étale algebras are equivalent.

For $n \geq 1$ we let $\mathrm{Q} \operatorname{Cov}_{\Gamma}^{n}$ denote the groupoid whose objects are thin $\Gamma$-quadrics of dimension $2(n-1)$, i.e., double coverings $Y_{0} \stackrel{\pi}{\leftarrow} Y$ of $\Gamma$-sets with $\left|Y_{0}\right|=n$, and whose morphisms are isomorphisms of $\Gamma$-coverings. Let QÉtex ${ }_{F}^{n}$ denote the 
groupoid of quadratic étale extensions $L / L_{0}$ of $F$-algebras with $\operatorname{dim}_{F} L_{0}=n$. From (2.3) we obtain an anti-equivalence of groupoids

$$
\operatorname{QÉtex~}_{F}^{n} \equiv \mathrm{QCov}_{\Gamma}^{n} \text {. }
$$

Isomorphism classes of these groupoids are in bijection with $H^{1}\left(\Gamma, \mathfrak{S}_{2}^{n} \rtimes \mathfrak{S}_{n}\right)$ (see [8]). We denote by $\mathbf{Y}\left(L / L_{0}\right)$ or $\mathbf{Y}(L, \sigma)$ the double covering (i.e., the thin $2(n-1)$-dimensional quadric) attached to the quadratic extension $L / L_{0}$ :

$$
\mathbf{Y}\left(L / L_{0}\right)=\mathbf{X}\left(L_{0}\right) \leftarrow \mathbf{X}(L), \quad \mathbf{Y}(L, \sigma)=\mathbf{X}(L) / \mathbf{X}(\sigma) \leftarrow \mathbf{X}(L)
$$

The following observation on symmetric and alternating elements in an étale algebra with involution will be used in the next subsection:

Proposition 3.5. For any étale F-algebra of even dimension with involution $(L, \sigma)$, we have

$$
\operatorname{Sym}(L, \sigma)=\operatorname{Symd}(L, \sigma) \quad \text { and } \quad \operatorname{Skew}(L, \sigma)=\operatorname{Alt}(L, \sigma) .
$$

Proof. The proposition is clear if char $F \neq 2$, for then

$$
x=\frac{x}{2}+\sigma_{L}\left(\frac{x}{2}\right) \quad \text { and } \quad y=\frac{y}{2}-\sigma_{L}\left(\frac{y}{2}\right) \quad \text { for } x \in \operatorname{Sym}(L, \sigma) \text { and } y \in \operatorname{Skew}(L, \sigma) .
$$

For the rest of the proof, we may thus assume char $F=2$. The linear map $L \rightarrow \operatorname{Symd}(L, \sigma)$ that carries $\ell \in L$ to $\ell+\sigma(\ell)$ fits into the exact sequence

$$
0 \rightarrow \operatorname{Sym}(L, \sigma) \rightarrow L \rightarrow \operatorname{Symd}(L, \sigma) \rightarrow 0 .
$$

Since $\operatorname{dim}_{F} \operatorname{Sym}(L, \sigma)=\frac{1}{2} \operatorname{dim}_{F} L$, we have $\operatorname{dim} \operatorname{Symd}(L, \sigma)=\operatorname{dim} \operatorname{Sym}(L, \sigma)$. It follows that the obvious inclusion $\operatorname{Symd}(L, \sigma) \subseteq \operatorname{Sym}(L, \sigma)$ is an equality, which proves the first equation. The second equation is the same as the first since $\operatorname{char} F=2$.

Embeddings of quadrics and of algebras with involution. Let $A$ be a central simple $F$-algebra of even degree $2 n$, and let $(\sigma, f)$ be a quadratic pair on $A$. If $\left(L, \sigma_{L}\right)$ is an étale $F$-algebra of dimension $2 n$ with involution, we define an embedding of algebras with involution

$$
\varepsilon:\left(L, \sigma_{L}\right) \hookrightarrow(A, \sigma, f)
$$

to be an $F$-algebra embedding $\varepsilon: L \hookrightarrow A$ satisfying the following conditions:

(a) $\varepsilon \circ \sigma_{L}=\sigma \circ \varepsilon$, and 
(b) $f(x)=\operatorname{Trd}_{A}(\varepsilon(\ell) x)$ for all $x \in \operatorname{Sym}(\sigma)$ and all $\ell \in L$ such that $\ell+\sigma_{L}(\ell)=$ 1.

Note that Proposition 3.5 shows that there exists $\ell \in L$ such that $\ell+\sigma_{L}(\ell)=1$, since $1 \in \operatorname{Sym}\left(\sigma_{L}\right)$. If $\ell, \ell^{\prime} \in L$ satisfy

$$
\ell+\sigma_{L}(\ell)=\ell^{\prime}+\sigma_{L}\left(\ell^{\prime}\right)=1,
$$

then $\ell-\ell^{\prime} \in \operatorname{Skew}\left(\sigma_{L}\right)$, and Proposition 3.5 yields $\ell-\ell^{\prime} \in \operatorname{Alt}\left(\sigma_{L}\right)$. If (a) holds, then $\varepsilon(\ell)-\varepsilon\left(\ell^{\prime}\right) \in \operatorname{Alt}(\sigma)$, hence, by $[7,(2.3)]$,

$$
\operatorname{Trd}_{A}\left(x\left(\varepsilon(\ell)-\varepsilon\left(\ell^{\prime}\right)\right)\right)=0 \quad \text { for all } x \in \operatorname{Sym}(\sigma) .
$$

Therefore, when (a) holds, condition (b) is equivalent to

(b') there exists $\ell \in L$ such that $\ell+\sigma_{L}(\ell)=1$ and $f(x)=\operatorname{Trd}_{A}(\varepsilon(\ell) x)$ for all $x \in \operatorname{Sym}(\sigma)$.

It can be further weakened to

(b") there exists $\ell \in L$ such that $f(x)=\operatorname{Trd}_{A}(\varepsilon(\ell) x)$ for all $x \in \operatorname{Sym}\left(\sigma_{L}\right)$,

because this condition on $\ell$ implies $\varepsilon(\ell)+\sigma \varepsilon(\ell)=1$, see $[7,(5.7)]$. Note that condition (b') (hence also (b")) holds with $\ell=\frac{1}{2}$ if $\operatorname{char} F \neq 2$.

Example 3.6. Let $\left(L, \sigma_{L}\right)$ be an arbitrary étale $F$-algebra with involution of the first kind. Let $L_{0}=\operatorname{Sym}\left(\sigma_{L}\right) \subset L$ be the fixed subalgebra under $\sigma_{L}$, let $T_{0}: L_{0} \rightarrow F$ be the trace, and let $t: L \rightarrow F$ be the quadratic form given by $t(x)=T_{0}(\sigma(x) x)$ for $x \in L$. Let $\left(\sigma_{t}, f_{t}\right)$ denote the adjoint quadratic pair on $\operatorname{End}_{F} L$. The regular representation is an embedding

$$
\left(L, \sigma_{L}\right) \hookrightarrow\left(\operatorname{End}_{F} L, \sigma_{t}, f_{t}\right) .
$$

Indeed, condition (a) holds because the polar form of $t$ is

$$
b_{t}(x, y)=T_{L_{0} / F}\left(\sigma_{L}(x) y+\sigma_{L}(y) x\right) \quad \text { for } x, y \in L,
$$

and we have

$$
b_{t}(x y, z)=b_{t}\left(y, \sigma_{L}(x) z\right) \quad \text { for } x, y, z \in L .
$$

Condition (b) also is easily checked, see [7, (5.12)]. 
Proposition 3.7. Use the same notation as in Example 3.6. Suppose L is split and let $(A, \sigma, f)$ be a central simple F-algebra with quadratic pair. For any embedding

$$
\varepsilon:\left(L, \sigma_{L}\right) \hookrightarrow(A, \sigma, f)
$$

there is an isomorphism $\varphi:(A, \sigma, f) \rightarrow\left(\operatorname{End}_{F} L, \sigma_{t}, f_{t}\right)$ such that $\varphi \circ \varepsilon:\left(L, \sigma_{L}\right) \hookrightarrow$ $\left(\operatorname{End}_{F} L, \sigma_{t}, f_{t}\right)$ is the regular representation.

Proof. Let $e_{1}, \ldots, e_{n}, e_{1}^{\prime}, \ldots, e_{n}^{\prime}$ be the collection of primitive idempotents of $L$, labelled in such a way that $e_{i}^{\prime}=\sigma_{L}\left(e_{i}\right)$ for $i \in \boldsymbol{n}$. As observed in the proof of Proposition 2.4, we may identify $A=\operatorname{End}_{F} V$ and we have a decomposition into 1-dimensional subspaces

$$
V=V_{1} \oplus \cdots \oplus V_{n} \oplus V_{1}^{\prime} \oplus \cdots \oplus V_{n}^{\prime}
$$

such that $\varepsilon\left(e_{i}\right)$ (resp. $\varepsilon\left(e_{i}^{\prime}\right)$ ) is the projection $V \rightarrow V_{i}$ (resp. $V \rightarrow V_{i}^{\prime}$ ). Let $q: V \rightarrow F$ be a quadratic form such that $(\sigma, f)$ is adjoint to $q$, and let $b$ be the polar form of $q$. Since $\sigma$ is adjoint to $b$ and $\sigma \varepsilon\left(e_{i}\right)=\varepsilon \sigma_{L}\left(e_{i}\right)=\varepsilon\left(e_{i}^{\prime}\right)$, we have for all $i, j \in \boldsymbol{n}$

$$
b\left(V_{i}, V_{j}\right)=b\left(\varepsilon\left(e_{i}\right)\left(V_{i}\right), V_{j}\right)=b\left(V_{i}, \varepsilon\left(e_{i}^{\prime}\right)\left(V_{j}\right)\right)=\{0\} .
$$

Similarly, $b\left(V_{i}^{\prime}, V_{j}^{\prime}\right)=\{0\}$ for all $i, j \in \boldsymbol{n}$, and $b\left(V_{i}, V_{j}^{\prime}\right)=\{0\}$ if $i \neq j$. Since $b$ is nonsingular, it follows that $b\left(V_{i}, V_{i}^{\prime}\right)=F$ for all $i \in \boldsymbol{n}$, hence we may find $v_{i} \in V_{i}$, $v_{i}^{\prime} \in V_{i}^{\prime}$ such that

$$
b\left(v_{i}, v_{i}^{\prime}\right)=1
$$

Let $S \in \operatorname{End}_{F} V$ be the map such that $S\left(v_{i}\right)=v_{i}^{\prime}$ and $S\left(v_{i}^{\prime}\right)=v_{i}$ for all $i \in \boldsymbol{n}$. It is readily verified that for $i \in \boldsymbol{n}$

$$
S \circ \varepsilon\left(e_{i}^{\prime}\right): x \mapsto v_{i} b\left(v_{i}, x\right) \quad \text { and } \quad S \circ \varepsilon\left(e_{i}\right): x \mapsto v_{i}^{\prime} b\left(v_{i}^{\prime}, x\right) \quad \text { for all } x \in V .
$$

Therefore, by [7, (5.11)],

$$
q\left(v_{i}\right)=f\left(S \circ \varepsilon\left(e_{i}^{\prime}\right)\right) \quad \text { and } \quad q\left(v_{i}^{\prime}\right)=f\left(S \circ \varepsilon\left(e_{i}\right)\right) .
$$

If $\ell \in L$ is such that $f(x)=\operatorname{Tr}(\varepsilon(\ell) \circ x)$ for all $x \in \operatorname{Sym}(\sigma)$, then

$$
f\left(S \circ \varepsilon\left(e_{i}^{\prime}\right)\right)=\operatorname{Tr}\left(\varepsilon(\ell) \circ S \circ \varepsilon\left(e_{i}^{\prime}\right)\right)=\operatorname{Tr}\left(S \circ \varepsilon\left(e_{i}^{\prime} \ell\right)\right) .
$$

None of the base vectors $v_{1}, \ldots, v_{n}^{\prime}$ is mapped to a nonzero multiple of itself by $S \circ \varepsilon\left(e_{i}^{\prime} \ell\right)$, hence $\operatorname{Tr}\left(S \circ \varepsilon\left(e_{i}^{\prime} \ell\right)\right)=0$ and therefore $q\left(v_{i}\right)=0$. Likewise, $q\left(v_{i}^{\prime}\right)=0$ for $i \in \boldsymbol{n}$. Therefore, mapping $v_{i} \mapsto e_{i}$ and $v_{i}^{\prime} \mapsto e_{i}^{\prime}$ for $i \in \boldsymbol{n}$ defines an isometry $(V, q) \rightarrow(L, t)$, and the induced isomorphism of algebras with quadratic pairs 
$\varphi:\left(\operatorname{End}_{F} V, \sigma, f\right) \rightarrow\left(\operatorname{End}_{F} L, \sigma_{t}, f_{t}\right)$ is such that $\varphi \circ \varepsilon$ is the regular representation.

We now turn to the geometric counterpart of the notion of embeddings of algebras with involution. Let $Q \subset P$ be a $\Gamma$-quadric of dimension $2(n-1)$ in a $\Gamma$-projective space of dimension $2 n-1$ over a field $F$. We let $C(Q)$ denote the $\Gamma$-set of $(n-1)$ dimensional linear varieties that lie in $Q$, and call this $\Gamma$-set the Clifford $\Gamma$-set of $Q$. If $(Y, \sigma)$ is a thin $\Gamma$-quadric of dimension $2(n-1)$, we define an embedding of quadrics

$$
\varepsilon:(Y, \sigma) \hookrightarrow Q
$$

as a general embedding $\varepsilon: Y \hookrightarrow P$ that carries isotropic linear subvarieties of $(Y, \sigma)$ to isotropic linear subvarieties of $P$, i.e., to linear subvarieties of $Q$. In particular, the image of $Y$ lies on $Q$. Since every isotropic linear subvariety is contained in a maximal isotropic linear subvariety, it suffices to require that the induced map $G r_{n-1} \varepsilon: G r_{n-1} Y \hookrightarrow G r_{n-1}(P)$ carry the set of maximal isotropic linear subvarieties $C(Y, \sigma)$ to $C(Q)$. We let $C(\varepsilon)$ denote the $\Gamma$-equivariant map induced by $G r_{n-1} \varepsilon$,

$$
C(\varepsilon): C(Y, \sigma) \hookrightarrow C(Q) .
$$

Let $\left(L, \sigma_{L}\right)$ be an arbitrary étale $F$-algebra of dimension $2 n$ with involution of the first kind, and let

$$
\varepsilon:\left(L, \sigma_{L}\right) \hookrightarrow(A, \sigma, f)
$$

be an embedding in a central simple $F$-algebra of degree $2 n$ with quadratic pair. Since $\varepsilon: L \hookrightarrow A$ is an $F$-algebra embedding, we may consider the general embedding of Proposition 2.6:

$$
\varepsilon_{*}: \mathbf{X}(L) \hookrightarrow \mathcal{S B}(A) .
$$

Let $\mathbf{Y}\left(L, \sigma_{L}\right)$ be the thin $\Gamma$-quadric associated with $\left(L, \sigma_{L}\right)$ and $\mathbf{Q}(\sigma, f)$ the $\Gamma$ quadric associated with $(A, \sigma, f)$.

Proposition 3.8. The map $\varepsilon_{*}$ defines an embedding of quadrics

$$
\varepsilon_{*}: \mathbf{Y}\left(L, \sigma_{L}\right) \hookrightarrow \mathbf{Q}(\sigma, f) .
$$

Proof. As usual, let $A_{s}=A \otimes_{F} F_{s}$, and let $\left(\sigma_{s}, f_{s}\right)$ be the quadratic pair on $A_{s}$ extending $(\sigma, f)$. We have to show that for every $\omega \in C\left(\mathbf{Y}\left(L, \sigma_{L}\right)\right)$ we have 
$G r_{n-1} \varepsilon_{*}(\omega) \in C(\mathbf{Q}(\sigma, f))$. Let $\xi, \eta \in \mathbf{X}(L)$. For $x \in \varepsilon_{*}(\xi), y \in \varepsilon_{*}(\eta)$ and $\ell \in L$ we have

$$
(1 \otimes \xi(\ell)) \cdot x=(\varepsilon(\ell) \otimes 1) \cdot x \quad \text { and } \quad(1 \otimes \eta(\ell)) \cdot y=(\varepsilon(\ell) \otimes 1) \cdot y,
$$

hence also

$$
\sigma_{s}(x) \cdot(\varepsilon(\ell) \otimes 1)=\sigma_{s}(x) \cdot\left(1 \otimes \xi \sigma_{L}(\ell)\right)
$$

and

$$
\sigma_{s}(x) \cdot y \cdot(1 \otimes \eta(\ell))=\sigma_{s}(x) \cdot(\varepsilon(\ell) \otimes 1) \cdot y=\sigma_{s}(x) \cdot y \cdot\left(1 \otimes \xi \sigma_{L}(\ell)\right) .
$$

If $\eta \neq \xi \sigma_{L}$, we can find $\ell \in L$ such that $\eta(\ell) \neq \xi \sigma_{L}(\ell)$, hence the last equation shows that $\sigma_{s}(x) \cdot y=0$. Therefore,

$$
\sigma_{s}\left(\varepsilon_{*}(\xi)\right) \cdot \varepsilon_{*}(\eta)=\{0\}, \quad \text { unless } \eta=\xi \sigma_{L} .
$$

In particular, for every $\omega \in C\left(\mathbf{Y}\left(L, \sigma_{L}\right)\right)$,

$$
\sigma_{s}\left(\bigoplus_{\xi \in \omega} \varepsilon_{*}(\xi)\right) \cdot \bigoplus_{\xi \in \omega} \varepsilon_{*}(\xi)=\{0\}, \quad \text { i.e., } \quad \sigma_{s}\left(G r_{n-1} \varepsilon_{*}(\omega)\right) \cdot G r_{n-1} \varepsilon_{*}(\omega)=\{0\} .
$$

Now, let $\ell \in L$ be such that $f(x)=\operatorname{Trd}(\varepsilon(\ell) x)$ for all $x \in \operatorname{Sym}(\sigma)$. For $x \in$ $G r_{n-1} \varepsilon_{*}(\omega) \cap \operatorname{Sym}\left(\sigma_{s}\right)$ we have

$$
f_{s}(x)=\operatorname{Tr}((\varepsilon(\ell) \otimes 1) \cdot x) \quad \text { since } x \in \operatorname{Sym}\left(\sigma_{s}\right),
$$

and

$$
(\varepsilon(\ell) \otimes 1) \cdot x \in G r_{n-1} \varepsilon_{*}(\omega) \quad \text { since } x \in G r_{n-1} \varepsilon_{*}(\omega) .
$$

On the other hand, we have $x=\sigma_{s}(x) \in \sigma_{s}\left(G r_{n-1} \varepsilon_{*}(\omega)\right)$, hence by (3.9),

$$
x \cdot(\varepsilon(\ell) \otimes 1) \cdot x=0 .
$$

It follows that $((\varepsilon(\ell) \otimes 1) \cdot x)^{2}=0$, hence $\operatorname{Tr}((\varepsilon(\ell) \otimes 1) \cdot x)=0$ and (3.10) shows that $f_{s}(x)=0$. Thus, $G r_{n-1} \varepsilon_{*}(\omega)$ is isotropic for all $\omega \in C\left(\mathbf{Y}\left(L, \sigma_{L}\right)\right), \mathrm{S}$ and the proposition is proved.

Conversely, every embedding

$$
\varepsilon:(Y, \varphi) \hookrightarrow \mathbf{Q}(\sigma, f)
$$

of a $\Gamma$-set $Y$ of $2 n$ elements with an involution $\varphi$ of the first kind in the quadric associated with a quadratic pair on a central simple algebra $A$ of degree $2 n$ arises from the embedding of the étale $F$-algebra with involution $\mathbf{M}(Y, \varphi)$ into $(A, \sigma, f)$, as we now show. 
Let $\varepsilon(Y)=\left\{I_{1}, \ldots, I_{n}, I_{1}^{\prime}, \ldots, I_{n}^{\prime}\right\}$, where the isotropic $2 n$-dimensional right ideals $I_{1}, \ldots, I_{n}^{\prime}$ of $A_{s}$ are indexed in such a way that $I_{i}^{\prime}=\varepsilon\left(\xi^{\varphi}\right)$ if $\varepsilon(\xi)=I_{i}$. As in $\S 2$, let

$$
L_{s}=\left\{x \in A_{s} \mid x \cdot I_{i} \subset I_{i} \text { and } x \cdot I_{i}^{\prime} \subset I_{i}^{\prime} \text { for all } i \in \boldsymbol{n}\right\}
$$

and $L=L_{s}^{\Gamma}$. Proposition 2.7 shows that we may identify $X$ with $\mathbf{X}(L)$ in such a way that $\varepsilon$ is the general embedding $\mathbf{X}(L) \hookrightarrow \mathcal{S B}(A)$ induced by the inclusion $L \hookrightarrow A$. Let $\sigma_{L}$ be the involution on $L$ such that $\mathbf{X}\left(\sigma_{L}\right)=\varphi$.

Proposition 3.11. The inclusion $L \hookrightarrow A$ is an embedding $\left(L, \sigma_{L}\right) \hookrightarrow(A, \sigma, f)$.

Proof. The primitive idempotents $e_{1}, \ldots, e_{n}, e_{1}^{\prime}, \ldots, e_{n}^{\prime}$ of $L_{s}$ are in one-to-one correspondence with $I_{1}, \ldots, I_{n}, I_{1}^{\prime}, \ldots, I_{n}^{\prime}$ in such a way that $I_{i}=e_{i} \cdot A_{s}$ and $I_{i}^{\prime}=e_{i}^{\prime} \cdot A_{s}$ for $i \in \boldsymbol{n}$. The indexing of $I_{1}, \ldots, I_{n}^{\prime}$ is such that $\sigma_{L_{s}}$ interchanges $e_{i}$ and $e_{i}^{\prime}$ for $i \in \boldsymbol{n}$. To check that $\sigma$ restricts to $\sigma_{L}$, observe that $I_{i}^{\prime}$ is the only element among $\left\{I_{1}, \ldots, I_{n}^{\prime}\right\}$ that does not belong to any $\omega^{C(\varepsilon)}$ for any $\omega \in C(X, *)$ containing $i$. Therefore,

$$
\sigma_{s}\left(I_{i}\right) \cdot I_{j}=\sigma_{s}\left(I_{i}\right) \cdot I_{k}^{\prime}=\{0\} \quad \text { for all } j, k \in \boldsymbol{n} \text { with } k \neq i,
$$

hence for all $i \in \boldsymbol{n}$

$$
\sigma_{s}\left(e_{i}\right) \cdot e_{j}=\sigma_{s}\left(e_{i}\right) \cdot e_{k}^{\prime}=0 \quad \text { for all } j, k \in \boldsymbol{n} \text { with } k \neq i .
$$

Similarly, for all $i \in \boldsymbol{n}$,

$$
\sigma_{s}\left(e_{i}^{\prime}\right) \cdot e_{j}=\sigma_{s}\left(e_{i}^{\prime}\right) \cdot e_{k}^{\prime}=0 \quad \text { for all } j, k \in \boldsymbol{n} \text { with } j \neq i .
$$

Since $e_{1}+\cdots+e_{n}+e_{1}^{\prime}+\cdots+e_{n}^{\prime}=1$, it follows that for all $i \in \boldsymbol{n}$ we have

$$
e_{i}^{\prime}=\left(\sum_{j \in \boldsymbol{n}} \sigma_{s}\left(e_{j}\right)+\sigma_{s}\left(e_{j}^{\prime}\right)\right) \cdot e_{i}^{\prime}=\sigma_{s}\left(e_{i}\right) \cdot e_{i}^{\prime}
$$

and, on the other hand,

$$
\sigma_{s}\left(e_{i}\right) \cdot\left(1-e_{i}^{\prime}\right)=\sigma_{s}\left(e_{i}\right) \cdot\left(\sum_{j \in \boldsymbol{n}} e_{j}+\sum_{k \in \boldsymbol{n} \backslash\{i\}} e_{k}^{\prime}\right)=0,
$$

hence $\sigma_{s}\left(e_{i}\right)=\sigma_{s}\left(e_{i}\right) \cdot e_{i}^{\prime}$. Therefore, $\sigma_{s}\left(e_{i}\right)=e_{i}^{\prime}$ for $i \in \boldsymbol{n}$.

To complete the proof that $\left(L, \sigma_{L}\right) \hookrightarrow(A, \sigma, f)$, it remains to show that for all $x \in \operatorname{Sym}(\sigma)$ and all $\ell \in L$ such that $\ell+\sigma_{L}(\ell)=1$ we have $f(x)=\operatorname{Trd}_{A}(\ell x)$. To show that this equation holds for all $x \in \operatorname{Sym}\left(\sigma_{s}\right)$ and all $\ell \in L_{s}$ such that $\ell+\sigma_{s}(\ell)=1$, it actually suffices to prove it for a single $\ell$, as was observed in the 
equivalence of conditions (b) and (b') defining an embedding. Let $\ell=\sum_{i \in \boldsymbol{n}} e_{i}$. Since $\ell+\sigma_{s}(\ell)=1$ we have for all $y \in A_{s}$

$$
\operatorname{Trd}_{A_{s}}\left(\ell \cdot\left(y+\sigma_{s}(y)\right)\right)=\operatorname{Trd}_{A_{s}}\left(\left(\ell+\sigma_{s}(\ell)\right) \cdot y\right)=\operatorname{Trd}_{A_{s}}(y) .
$$

Since $f_{s}\left(y+\sigma_{s}(y)\right)=\operatorname{Trd}_{A_{s}}(y)$, we have

$$
f_{s}(x)=\operatorname{Trd}_{A_{s}}(\ell x) \quad \text { for all } x \in \operatorname{Symd}\left(\sigma_{s}\right) .
$$

Let $I=\ell \cdot A_{s}=I_{1}+\cdots+I_{n}$ and $I^{\prime}=(1-\ell) \cdot A_{s}=I_{1}^{\prime}+\cdots+I_{n}^{\prime}$. These right ideals are isotropic since $\left\{I_{1}, \ldots, I_{n}\right\},\left\{I_{1}^{\prime}, \ldots, I_{n}^{\prime}\right\} \in C(\varepsilon(X, *))$. For $x \in I \cap \operatorname{Sym}\left(\sigma_{s}\right)$ we have $f_{s}(x)=0$ and, on the other hand, $\ell x \in I$ and $x \ell x \in \sigma_{s}(I) \cdot I=\{0\}$, hence $(\ell x)^{2}=0$. Therefore,

$$
f_{s}(x)=\operatorname{Trd}_{A_{s}}(\ell x) \quad \text { for all } x \in I \cap \operatorname{Sym}\left(\sigma_{s}\right) .
$$

Similarly, for $x \in I^{\prime} \cap \operatorname{Sym}\left(\sigma_{s}\right)$ we have $\ell x=0$, hence

$$
f_{s}(x)=\operatorname{Trd}_{A_{s}}(\ell x) \quad \text { for all } x \in I^{\prime} \cap \operatorname{Sym}\left(\sigma_{s}\right) .
$$

Now, for $x \in \operatorname{Sym}\left(\sigma_{s}\right)$ we have

$$
x=\ell x(1-\ell)+(1-\ell) x \ell+\ell x \ell+(1-\ell) x(1-\ell) .
$$

Since $\ell \in I, 1-\ell \in I^{\prime}$, and $\sigma_{s}(\ell)=1-\ell$, we have

$$
\ell x(1-\ell) \in I \cap \operatorname{Sym}\left(\sigma_{s}\right), \quad(1-\ell) x \ell \in I^{\prime} \cap \operatorname{Sym}\left(\sigma_{s}\right),
$$

and

$$
\ell x \ell+(1-\ell) x(1-\ell)=\ell x \ell+\sigma_{s}(\ell x \ell) \in \operatorname{Symd}\left(\sigma_{s}\right) .
$$

Therefore,

$$
f_{s}(x)=f_{s}(\ell x(1-\ell))+f_{s}((1-\ell) x \ell)+f_{s}(\ell x \ell+(1-\ell) x(1-\ell)),
$$

and it follows from (3.12), (3.13), and (3.14) that

$$
f_{s}(x)=\operatorname{Trd}_{A_{s}}(\ell x) .
$$

\section{Clifford algebras and $\Gamma$-Sets}

As in the previous sections, we let $F$ denote an arbitrary field and $\Gamma$ the Galois group of a separable closure $F_{s}$ of $F$. 
Clifford constructions. Recall from $\S 3$ that for any $2(n-1)$-dimensional thin $\Gamma$-quadric $Y / Y_{0}$ we let $C\left(Y / Y_{0}\right)$ denote the $\Gamma$-set of maximal linear isotropic subvarieties, which can be identified with the $2^{n}$-element set of sections of the covering $Y_{0} \leftarrow Y$. We call $C\left(Y / Y_{0}\right)$ the Clifford $\Gamma$-set of $Y / Y_{0}$. The canonical involution $\sigma$ of $Y / Y_{0}$ induces an involution $\underline{\sigma}$ on $C\left(Y / Y_{0}\right)$, which maps every section to its complement in $Y$. If $n=1$, then $C\left(Y / Y_{0}\right)=Y$ and $\underline{\sigma}=\sigma$. The following proposition is clear:

Proposition 4.1. If $Y / Y_{0}$ and $Z / Z_{0}$ are thin $\Gamma$-quadrics, then $(Y \sqcup Z) /\left(Y_{0} \sqcup Z_{0}\right)$ is a thin $\Gamma$-quadric and there is a canonical isomorphism

$$
C\left(Y / Y_{0}\right) \times C\left(Z / Z_{0}\right) \stackrel{\sim}{\longrightarrow} C\left(Y \sqcup Z / Y_{0} \sqcup Z_{0}\right)
$$

which maps any pair of isotropic linear varieties $\left(\omega, \omega^{\prime}\right)$ to $\omega \sqcup \omega^{\prime}$.

We now turn to the analogue of this construction for quadratic étale extensions. Let $\varepsilon: L_{0} \hookrightarrow L$ be a quadratic étale extension of étale $F$-algebras, with $\operatorname{dim}_{F} L_{0}=n$. In view of the Grothendieck correspondence there exists an étale algebra $C\left(L / L_{0}\right)$ of dimension $2^{n}$, with involution $\underline{\sigma}$, such that $C \mathbf{Y}\left(L / L_{0}\right)=$ $\mathbf{Y}\left(C\left(L / L_{0}\right), \underline{\sigma}\right)$. This étale algebra is constructed explicitly in [8], where it is designated by $\Omega\left(L / L_{0}\right)$. We briefly recall the construction: the algebra $L_{0}^{\otimes n}$ contains an idempotent $e_{n}^{L_{0}}$ that corresponds under the composition of canonical isomorphisms

$$
L_{0}^{\otimes n} \stackrel{\sim}{\longrightarrow} \mathbf{M}\left(\mathbf{X}\left(L_{0}^{\otimes n}\right)\right) \stackrel{\sim}{\longrightarrow} \mathbf{M}\left(\mathbf{X}\left(L_{0}\right)^{n}\right)
$$

to the characteristic function $\chi$ of the complement of the "fat diagonal" of $\mathbf{X}\left(L_{0}\right)^{n}$ :

$$
\chi\left(\xi_{1}, \ldots, \xi_{n}\right)= \begin{cases}1 & \text { if } \xi_{1}, \ldots, \xi_{n} \text { are all different; } \\ 0 & \text { if } \xi_{i}=\xi_{j} \text { for some } i, j \text { with } i \neq j .\end{cases}
$$

Then, letting $\left(L^{\otimes n}\right)^{\mathfrak{S}_{n}}$ denote the subalgebra of $L^{\otimes n}$ fixed under the action of $\mathfrak{S}_{n}$ by permutation of the factors, we set

$$
C\left(L / L_{0}\right)=\varepsilon^{\otimes n}\left(e_{n}^{L_{0}}\right) \cdot\left(L^{\otimes n}\right)^{\mathfrak{S}_{n}} .
$$

We call the algebra $C\left(L / L_{0}\right)$ the Clifford algebra of $L / L_{0}$. If $n=1$, then $C\left(L / L_{0}\right)=L$, and the canonical involution $\underline{\sigma}$ is the canonical involution of $L / L_{0}$.

Proposition 4.2. If $L / L_{0}$ and $M / M_{0}$ are quadratic étale extensions, there is a canonical isomorphism

$$
P: C\left(L \times M / L_{0} \times M_{0}\right) \stackrel{\sim}{\longrightarrow} C\left(L / L_{0}\right) \otimes C\left(M / M_{0}\right),
$$


which corresponds under the Grothendieck correspondence to the canonical isomorphism of Proposition 4.1.

By contrast to $\Gamma$-sets, we have an $F$-linear map

$$
c_{L}: L \rightarrow C\left(L / L_{0}\right)
$$

defined by

$$
c_{L}(x)=\varepsilon^{\otimes n}\left(e_{n}^{L_{0}}\right) \cdot((x \otimes 1 \otimes \cdots \otimes 1)+(1 \otimes x \otimes \cdots \otimes 1)+(1 \otimes \cdots \otimes 1 \otimes x))
$$

for $x \in L$.

Proposition 4.4. Let $L / L_{0}$ and $M / M_{0}$ be quadratic étale extensions and let $\rho: L \times M \rightarrow L \otimes M$ be the linear map given by $\rho(x, y)=x \otimes 1+1 \otimes y$. The diagram

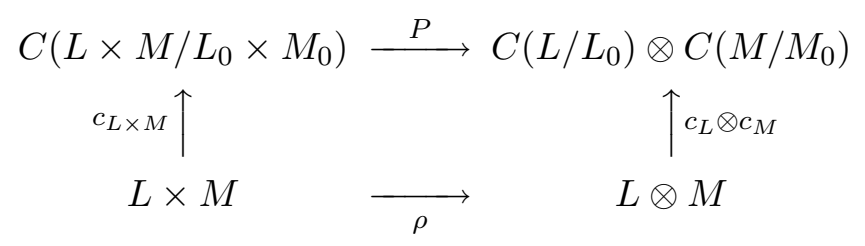

is commutative.

Proof. The claim follows easily from the definition of the canonical map $P$.

Corollary 4.5. The space $c_{L}(L)$ generates $C\left(L / L_{0}\right)$ as an algebra.

Proof. By extending $F$ we may assume that $L / L_{0}$ has a nontrivial decomposition $L / L_{0}=M / M_{0} \times N / N_{0}$. The claim then follows from (4.4) by induction on the dimension.

Lemma 4.6. Let $L_{0}=\operatorname{Sym}\left(\sigma_{L}\right)$ and $T_{0}: L_{0} \rightarrow F$ be the trace map. The kernel of $c_{L}: L \rightarrow C\left(L, \sigma_{L}\right)$ is

$$
\operatorname{ker} c_{L}=\left\{x \in L_{0} \mid T_{0}(x)=0\right\} \text {. }
$$

Proof. Extend scalars to $F_{s}$ and use induction on $\operatorname{dim} L$. 
Discriminants. Let $Q$ be a $2(n-1)$-dimensional $\Gamma$-quadric. The Clifford $\Gamma$ set $C(Q)$, consisting of maximal linear subvarieties of $Q$, decomposes into two classes $C_{1}(Q), C_{2}(Q)$ : two linear varieties $v_{1}, v_{2}$ lie in the same class if and only if $\operatorname{dim}\left(v_{1} \cap v_{2}\right) \equiv n-1 \bmod 2$, see for example [3, $\left.\S \S I I .6\right]$. The equivalence relation is clearly preserved under the action of $\Gamma$, hence $\Gamma$ acts on the set

$$
\Delta(Q):=\left\{C^{1}(Q), C^{2}(Q)\right\}
$$

which we call the discriminant of $Q$. We let $\delta: C(Q) \rightarrow \Delta(Q)$ denote the $\Gamma$ equivariant map that carries each linear variety in $C(Q)$ to its class.

Likewise, if $(Y, \sigma)$ is a thin $\Gamma$-quadric of dimension $2(n-1)$, the Clifford $\Gamma$-set $C(Y, \sigma)$ of $(n-1)$-dimensional isotropic linear subvarieties decomposes into two classes as for "classical" quadrics, hence we also have a discriminant $\Delta(Y, \sigma)$ and a canonical map $\delta: C(Y, \sigma) \rightarrow \Delta(Y, \sigma)$. An alternative description of this discriminant is given in [9, Proposition 2.5]: it is canonically isomorphic to the set of orbits under the alternating group $\mathfrak{A}_{2 n}$ of the set

$$
\Sigma_{2 n}(Y)=\left\{\left(y_{1}, \ldots, y_{2 n}\right) \mid Y=\left\{y_{1}, \ldots, y_{2 n}\right\}\right\} .
$$

Therefore, $\Delta(Y, \sigma)$ depends only on $Y$, and not on the involution $\sigma$; we may thus use the notation $\Delta(Y)$ for $\Delta(Y, \sigma)$.

Proposition 4.7. Let $\varepsilon:(Y, \sigma) \hookrightarrow Q$ be an embedding of quadrics. There is an induced isomorphism of $\Gamma$-sets $\Delta(\varepsilon): \Delta(Y) \rightarrow \Delta(Q)$ that fits into the following commutative diagram:

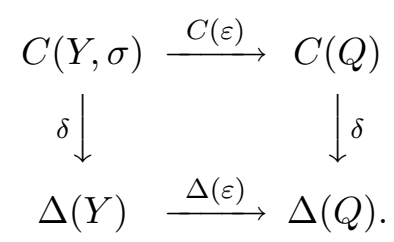

Proof. For $\omega_{1}, \omega_{2} \in C(Y, \sigma)$, we have

$$
\left|\omega_{1} \cap \omega_{2}\right|=\operatorname{dim}\left(C(\varepsilon)\left(\omega_{1}\right) \cap C(\varepsilon)\left(\omega_{2}\right)\right),
$$

hence the map $C(\varepsilon)$ is compatible with the division of $C(Y, \sigma)$ and $C(Q)$ into two classes. Therefore, we may define $\Delta(\varepsilon)$ by mapping the equivalence class of $\omega \in C(Y, \sigma)$ to the equivalence class of $C(\varepsilon)(\omega) \in C(Q)$. 
Under the Grothendieck correspondence, the discriminant of $\Gamma$-sets yields a discriminant of étale algebras, see $[8, \S 18]$. If $(L, \sigma)$ is an étale algebra with involution, we have a canonical map

$$
\delta: \Delta(L) \rightarrow C(L, \sigma)
$$

so we may consider the Clifford algebra $C(L, \sigma)$ as an étale extension of $\Delta(L)$.

Clifford algebra embeddings. Let $(A, \sigma, f)$ be a central simple algebra of even degree $2 n$ with quadratic pair over an arbitrary field $F$. Recall from $[7, \S 8]$ that the Clifford algebra $C(A, \sigma, f)$ is defined in such a way that if $A=\operatorname{End}_{F} V$ and $(\sigma, f)$ is induced by some quadratic form $q: V \rightarrow F$, then $C(A, \sigma, f)$ is the even Clifford algebra

$$
C(A, \sigma, f)=C_{0}(V, q)
$$

The center of $C(A, \sigma, f)$ is a quadratic étale $F$-algebra $Z(A, \sigma, f)$, which is related to the discriminant of $(\sigma, f)$, see $[7,(8.10)]$, and $C(A, \sigma, f)$ is an Azumaya algebra of constant rank $2^{n}$ over $Z(A, \sigma, f)$. The Clifford algebra $C(A, \sigma, f)$ is defined as a quotient of the tensor algebra $T(A)$ of the underlying vector space of $A$, hence there is a canonical $F$-linear map

$$
c_{A}: A \rightarrow C(A, \sigma, f) .
$$

In the split case $A=\operatorname{End}_{F} V$, the bilinear polar form of $q$ yields an $F$-linear isomorphism $V \rightarrow V^{*}$ which allows us to identify

$$
V \otimes_{F} V=V \otimes_{F} V^{*}=\operatorname{End}_{F} V
$$

under this identification, the map $c_{A}$ carries $v \otimes w \in V \otimes_{F} V$ to $v \cdot w \in C_{0}(V, q)$. Now, let $\operatorname{deg} A=2 n$, and let

$$
\varepsilon:\left(L, \sigma_{L}\right) \hookrightarrow(A, \sigma, f)
$$

be an embedding of an étale $F$-algebra with involution of the first kind of dimension $2 n$ in $(A, \sigma, f)$.

Theorem 4.8. There is an F-algebra embedding

$$
C(\varepsilon): C\left(L, \sigma_{L}\right) \hookrightarrow C(A, \sigma, f)
$$


such that the following diagram commutes:

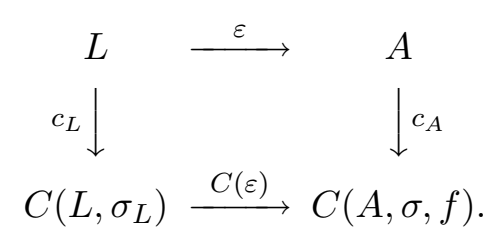

The F-algebra homomorphism $C(\varepsilon)$ is uniquely determined by this property. Moreover, the following diagram commutes:

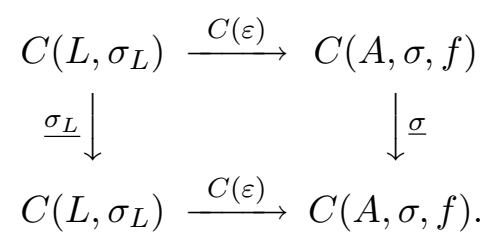

Furthermore, there is an F-algebra isomorphism $\overline{C(\varepsilon)}$ which makes the following diagram commute:

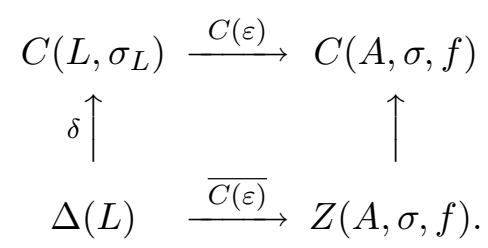

The proof uses the following general principle:

Principle 4.12. Let $C$ and $C^{\prime}$ be F-algebras, and let $U \subset C$ be a subspace which generates $C$ as an F-algebra. Let also $f: U \rightarrow C^{\prime}$ be a linear map. If there exists a field extension $K / F$ and a $K$-algebra homomorphism $\hat{f}_{K}: C \otimes_{F} K \rightarrow C^{\prime} \otimes_{F} K$ such that $\left.\hat{f}_{K}\right|_{U \otimes K}=f \otimes \operatorname{Id}_{K}$, then $f$ extends to an F-algebra homomorphism $\hat{f}: C \rightarrow C^{\prime}$.

Proof. The restriction of $\hat{f}_{K}$ to $C$ is an $F$-algebra homomorphism $C \rightarrow C^{\prime} \otimes_{F} K$. Since the restriction of $\hat{f}_{K}$ to $U$ is $f$, and $U$ generates $C$, the image of this homomorphism is in fact in $C^{\prime}$.

Proof of Theorem 4.8. Since $c(L)$ generates the algebra $C\left(L, \sigma_{L}\right)$, it is clear that, if it exists, $C(\varepsilon)$ is uniquely determined by the commutativity of diagram (4.10). By $[7,(8.14)]$, we have

$$
\operatorname{ker}\left(c_{A} \circ \varepsilon\right)=\{x \in L \mid \varepsilon(x) \in \operatorname{Sym}(\sigma) \text { and } f(\varepsilon(x))=0\} .
$$


Since $\varepsilon$ embeds $\left(L, \sigma_{L}\right)$ in $(A, \sigma, f)$, the condition $\varepsilon(x) \in \operatorname{Sym}\left(\sigma_{L}\right)$ holds if and only if $x \in L_{0}$, and then

$$
f(\varepsilon(x))=\operatorname{Trd}_{A}(\varepsilon(\ell x))=T(\ell x) \quad \text { for any } \ell \in L \text { such that } \ell+\sigma_{L}(\ell)=1 .
$$

Since for $x \in L_{0}$ we have

$$
T(\ell x)=T_{0}\left(\ell x+\sigma_{L}(\ell x)\right)=T_{0}(x)
$$

it follows from Lemma 4.6 that $\operatorname{ker} c_{L}=\operatorname{ker}\left(c_{A} \circ \varepsilon\right)$. Therefore, $\varepsilon$ induces an injective $F$-linear map $\varepsilon^{\prime}: c_{L}(L) \hookrightarrow C(A, \sigma, f)$. Since $c_{L}(L)$ generates $C\left(L, \sigma_{L}\right)$, we may apply the general principle above with $U=c_{L}(L), C=C\left(L, \sigma_{L}\right)$, and $C^{\prime}=C(A, \sigma, f)$, taking for $K$ a separable closure $F_{s}$ of $F$. To prove the existence of $C(\varepsilon)$, it suffices to show that $\varepsilon_{s}^{\prime}:=\varepsilon \otimes \operatorname{Id}_{F_{s}}$ extends to an $F_{s}$-algebra embedding

$$
C\left(\varepsilon_{s}\right): C\left(L_{s}, \sigma_{L_{s}}\right) \rightarrow C\left(A_{s}, \sigma_{s}, f_{s}\right)
$$

By Proposition 3.7, we may assume $A_{s}=\operatorname{End}_{F_{s}}\left(L_{s}\right),\left(\sigma_{s}, f_{s}\right)$ is the quadratic pair adjoint to the quadratic form $t: L_{s} \rightarrow F_{s}$ of Example 3.6, and $\varepsilon_{s}$ is the regular representation, hence $C\left(A_{s}, \sigma_{s}, f_{s}\right)=C_{0}\left(L_{s}, t\right)$.

We argue by induction on $n$. If $n=1$ (i.e. $L_{s}=F_{s} \times F_{s}$ ) the map $c_{L}$ is the identity

$$
c_{L}: L_{s} \stackrel{\sim}{\longrightarrow} C\left(L_{s}, \sigma_{L_{s}}\right)=L_{s},
$$

and $c_{A} \circ \varepsilon_{s}: L_{s} \rightarrow C_{0}\left(L_{s}, t\right)$ is an $F_{s}$-algebra isomorphism, so the result is clear.

If $n>1$ we may find a nontrivial decomposition $\left(L_{s}, \sigma_{s}\right)=\left(L_{1}, \sigma_{1}\right) \times\left(L_{2}, \sigma_{2}\right)$. We then have, by Proposition 4.2,

$$
C\left(L_{s}, \sigma_{s}\right)=C\left(L_{1}, \sigma_{1}\right) \otimes_{F} C\left(L_{2}, \sigma_{2}\right),
$$

and, letting $c_{i}: L \rightarrow C\left(L_{i}, \sigma_{i}\right)$ for $i=1,2$ denote the canonical $F_{s}$-linear map,

$$
c_{L}\left(\ell_{1}, \ell_{2}\right)=c_{1}\left(\ell_{1}\right) \otimes c_{2}\left(\ell_{2}\right) \quad \text { for } \ell_{1} \in L_{1} \text { and } \ell_{2} \in L_{2} .
$$

On the other hand, defining quadratic forms $t_{1}$ and $t_{2}$ on $L_{1}$ and $L_{2}$ respectively in the same way as $t$ on $L_{s}$, we have an orthogonal sum decomposition

$$
\left(L_{s}, t\right)=\left(L_{1}, t_{1}\right) \stackrel{\perp}{\bigoplus}\left(L_{2}, t_{2}\right)
$$

hence a canonical $F$-algebra embedding

$$
C_{0}\left(L_{1}, t_{1}\right) \otimes_{F} C_{0}\left(L_{2}, t_{2}\right) \hookrightarrow C_{0}\left(L_{s}, t\right) .
$$


By induction, we may assume $(c \circ \varepsilon)_{1}: L_{1} \rightarrow C_{0}\left(L_{1}, t_{1}\right)$ and $(c \circ \varepsilon)_{2}: L_{2} \rightarrow$ $C_{0}\left(L_{2}, t_{2}\right)$ extend to $F_{s}$-algebra embeddings

$$
C\left(\varepsilon_{1}\right): C\left(L_{1}, \sigma_{1}\right) \hookrightarrow C_{0}\left(L_{1}, t_{1}\right) \quad \text { and } \quad C\left(\varepsilon_{2}\right): C\left(L_{2}, \sigma_{2}\right) \hookrightarrow C_{0}\left(L_{2}, t_{2}\right) .
$$

Then the composition

$$
\begin{array}{rl}
C\left(L_{s}, \sigma_{L_{s}}\right)=C\left(L_{1}, \sigma_{1}\right) \otimes_{F} & C\left(L_{2}, \sigma_{2}\right) \\
& \stackrel{C\left(\varepsilon_{1}\right) \otimes C\left(\varepsilon_{2}\right)}{\longrightarrow} C_{0}\left(L_{1}, t_{1}\right) \otimes_{F} C_{0}\left(L_{2}, t_{2}\right) \hookrightarrow C_{0}\left(L_{s}, t\right)
\end{array}
$$

is an $F_{s}$-algebra embedding that extends $c_{A} \circ \varepsilon_{s}$.

To prove that diagram (4.11) commutes, it suffices to prove the commutativity of

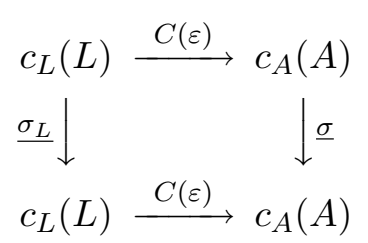

since $c_{L}(L)$ generates $C\left(L, \sigma_{L}\right)$. Commutativity of the latter diagram follows from commutativity of the diagrams

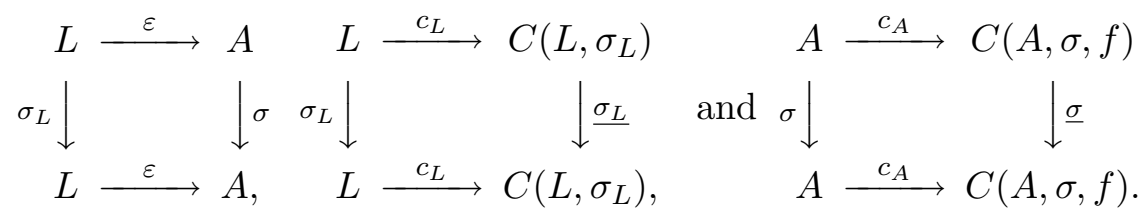

To complete the proof, it remains to show that $C(\varepsilon)$ maps $\Delta(L)$ to $Z(A, \sigma, f)$. It suffices to see that the image of $\Delta(L)$ centralizes $c_{A}(A)$ since $c_{A}(A)$ generates $C(A, \sigma, f)$. Again, we may extend scalars to reduce to the case where $L$ is split, and use induction on $\operatorname{dim} L$. Details are left to the reader.

Remark 4.13. The situation described in Theorem 4.8 is related to results about Cartan subspaces given in [17]. Assume that $F$ has characteristic different from 2. A Cartan subspace of a central simple algebra $A$ of even degree $2 n$ with an orthogonal involution $\sigma$ is by definition a subspace of $\operatorname{Skew}\left(A, \sigma_{A}\right)$ such that there exists an element $a \in H$ which is invertible in $A$, is separable (i.e., generates an étale $F$-subalgebra of $A$ ) and such that $a^{-1} H$ is an étale commutative subalgebra of $A$ of dimension $2 n$. Equivalently, if $a \in \operatorname{Skew}\left(A, \sigma_{A}\right)$ is invertible and separable in $A$, then $H=\left\{x \in \operatorname{Skew}\left(A, \sigma_{A}\right) \mid x a=a x\right\}$ is a Cartan subspace for $a$. It can be shown that the algebra $L_{0}=a^{-1} H$ is independent of the choice of the element 
a. Moreover the subalgebra $L=L_{0} \oplus H$ of $A$ is maximal commutative étale in $A$ and has an involution $\sigma_{L}$ which is the restriction of $\sigma_{A}$ to $L$. Thus the set of skew-symmetric elements in $L$ is a Cartan subspace of A. It follows from [17, Théorème 3] that $c_{A}(L)$ generates a maximal commutative étale subalgebra of $C\left(A, \sigma_{A}\right)$. This algebra is isomorphic to $C\left(L, \sigma_{L}\right)$.

The Severi-Brauer variety of a Clifford algebra. We discuss in this subsection the geometric analogue of Theorem 4.8, showing that an embedding of a thin quadric into a quadric induces an embedding of the corresponding Clifford $\Gamma$-sets.

Recall that the Severi-Brauer variety of a central simple algebra is a $\Gamma$-projective space. To take in account the fact that $C(A, \sigma, f)$ is an Azumaya algebra of constant rank over the étale $F$-algebra $Z(A, \sigma, f)$ we introduce a variant of the Severi-Brauer variety for Azumaya algebras over quadratic étale algebras." We consider disjoint unions $P_{1} \sqcup P_{2}$ of two projective $F$-spaces of the same dimension, endowed with a continuous action of a profinite group $\Gamma$ by collineations that may interchange the two components. We call these unions double $\Gamma$-projective spaces. They are equipped with a canonical structure map to a $\Gamma$-set $Z$ of two elements

$$
Z:=\left\{\left\{P_{1}\right\},\left\{P_{2}\right\}\right\} \leftarrow P_{1} \sqcup P_{2} .
$$

If $\operatorname{dim} P_{1}=\operatorname{dim} P_{2}=n-1$, we may define for every integer $k \in \boldsymbol{n}$ two different sets:

$$
\begin{aligned}
& g r_{k-1}\left(P_{1} \sqcup P_{2}\right)=G r_{k-1}\left(P_{1}\right) \cup G r_{k-1}\left(P_{2}\right) \quad \text { and } \\
& \qquad r_{k-1}\left(P_{1} \sqcup P_{2}\right)=\left\{v_{1} \sqcup v_{2} \mid v_{1} \in G r_{k-1}\left(P_{1}\right), v_{2} \in G r_{k-1}\left(P_{2}\right)\right\} .
\end{aligned}
$$

Note that, even though $G r_{k-1}\left(P_{1}\right)$ and $G r_{k-1}\left(P_{2}\right)$ are not $\Gamma$-sets since they may be interchanged under the $\Gamma$-action, $\Gamma$ naturally acts on $g r_{k-1}\left(P_{1} \sqcup P_{2}\right)$ and $G r_{k-1}\left(P_{1} \sqcup P_{2}\right)$, and $g r_{k-1}\left(P_{1} \sqcup P_{2}\right)$ carries a canonical $\Gamma$-equivariant map

$$
Z \leftarrow g r_{k-1}\left(P_{1} \sqcup P_{2}\right) .
$$

If $\Gamma_{0} \subset \Gamma$ is the subgroup fixing $Z$, we clearly have an isomorphism of $\Gamma_{0}$-sets

$$
G r_{k-1}\left(P_{1} \sqcup P_{2}\right)=G r_{k-1}\left(P_{1}\right) \times G r_{k-1}\left(P_{2}\right) .
$$

If $\Gamma_{0} \neq \Gamma$, then $G r_{k-1}\left(P_{1} \sqcup P_{2}\right)$ may be identified with the norm of the $\Gamma_{0}$-set $G r_{k-1}\left(P_{1}\right)$ (or $G r_{k-1}\left(P_{2}\right)$ ) following Ferrand's general definition in [4].

\footnotetext{
${ }^{\|}$For the sake of simplicity we give an ad-hoc construction.
} 
For $(Y \stackrel{\pi}{\leftarrow} X)$ a $\Gamma$-covering with $|Y|=2$ and $|X|=2 n$, a general embedding

$$
\varepsilon:(Y \stackrel{\pi}{\leftarrow} X) \hookrightarrow\left(Z \leftarrow P_{1} \sqcup P_{2}\right)
$$

is a pair of $\Gamma$-equivariant maps $\varepsilon: X \rightarrow g r_{0}\left(P_{1} \sqcup P_{2}\right), \bar{\varepsilon}: Y \rightarrow Z$ such that

(a) the following diagram commutes:

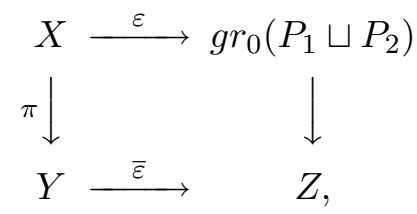

(b) $\bar{\varepsilon}$ is bijective, and

(c) the image of each fiber of $Y$ is a set of $n$ points in general position in $P_{1}$ and $P_{2}$ respectively.

The embedding $\varepsilon$ thus induces $\Gamma$-equivariant maps

$$
G r_{k-1} \varepsilon: G r_{k-1}(X / Y) \hookrightarrow g r_{k-1}\left(P_{1} \sqcup P_{2}\right) \quad \text { for all } k \in \boldsymbol{n} .
$$

These maps fit into commutative diagrams

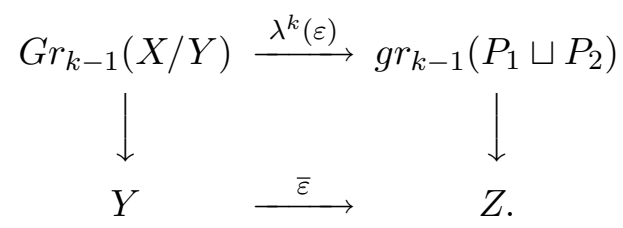

Double $\Gamma$-projective space arise from the following construction. Let $Z$ be a quadratic étale algebra over an arbitrary field $F$, and let $B$ be an Azumaya $Z$ algebra of constant rank. As usual, let $F_{s}$ denote a separable closure of $F$, let $Z_{s}=$ $Z \otimes_{F} F_{s}$ and $B_{s}=B \otimes_{F} F_{s}$ the $F_{s}$-algebras obtained by scalar extension, and let $\Gamma$ be the Galois group of $F_{s} / F$. If $\operatorname{dim}_{F} B=2 n^{2}$, then $B_{s} \simeq M_{n}\left(F_{s}\right) \times M_{n}\left(F_{s}\right)$; its minimal right ideals have dimension $n$. We let $\mathbb{S B}(B)$ be the double $\Gamma$-projective space whose points are the $n$-dimensional right ideals of $B_{s}$, with the natural $\Gamma$-action. These ideals are divided into two classes, which correspond to the two primitive idempotents of $Z_{s}$ : to each $n$-dimensional right ideal $I$, we associate the primitive idempotent $e_{I} \in Z_{s}$ such that $e_{I} \cdot I=I$. Alternatively, we associate to $I$ the element $I^{\delta} \in \mathbf{X}(Z)$ such that $I^{\delta}\left(e_{I}\right)=1$. Thus, the canonical structure map of the double $\Gamma$-projective space $\mathbb{S B}(B)$ can be identified with

$$
\mathbf{X}(Z) \stackrel{\delta}{\leftarrow} \mathbb{S B}(B) .
$$


Now, let $K \stackrel{i}{\rightarrow} L$ be an étale extension of degree $n$ of a quadratic étale $F$-algebra. (Thus, $\operatorname{dim}_{F} L=2 n$.) By definition, an embedding

$$
\varepsilon:(L / K) \hookrightarrow(B / Z)
$$

is an $F$-algebra embedding $L \hookrightarrow B$ that restricts to an isomorphism $\bar{\varepsilon}: K \stackrel{\sim}{\longrightarrow} Z$.

Proposition 4.14. If, in the situation above, $L$ is a split étale $F$-algebra, then for every embedding $\varepsilon:(L / K) \hookrightarrow(B / Z)$ there is an F-algebra isomorphism $\varphi: B \stackrel{\sim}{\longrightarrow}$ $\operatorname{End}_{K} L$ whose restriction to $Z$ is $\bar{\varepsilon}^{-1}$ such that $\varphi \circ \varepsilon: L \hookrightarrow \operatorname{End}_{K} L$ is the regular representation.

Proof. By hypothesis $K$ is split, hence $Z$ also. Using $\bar{\varepsilon}$ as an identification, let

$$
K=Z=F \times F,
$$

and consider the corresponding decompositions $L=L_{1} \times L_{2}, B=B_{1} \times B_{2}$, and $\varepsilon=\left(\varepsilon_{1}, \varepsilon_{2}\right)$. By Proposition 2.4, we may find $F$-algebra isomorphisms $\varphi_{i}: B_{i} \stackrel{\sim}{\longrightarrow} \operatorname{End}_{F} L_{i}$, for $i=1,2$, such that $\varphi_{i} \circ \varepsilon_{i}: L_{i} \hookrightarrow \operatorname{End}_{F} L_{i}$ is the regular representation. The proposition holds with $\varphi=\left(\varphi_{1}, \varphi_{2}\right): B \rightarrow \operatorname{End}_{F} L_{1} \times \operatorname{End}_{F} L_{2}=$ $\operatorname{End}_{K} L$.

Now, consider an arbitrary embedding $\varepsilon:(L / K) \hookrightarrow(B / Z)$ of an étale extension of degree $n$. For every $\xi \in \mathbf{X}(L)$ we let as in(2.5)

$$
\varepsilon_{*}(\xi)=\left\{x \in B_{s} \mid(1 \otimes \xi(\ell)) \cdot x=(\varepsilon(\ell) \otimes 1) \cdot x \text { for all } \ell \in L\right\} .
$$

Proposition 4.15. The right ideal $\varepsilon_{*}(\xi)$ is n-dimensional, and $\varepsilon_{*}$ defines a general embedding

$$
\varepsilon_{*}:(\mathbf{X}(K) \stackrel{\mathbf{X}(i)}{\longleftarrow} \mathbf{X}(L)) \hookrightarrow(\mathbf{X}(Z) \leftarrow \mathbb{S B}(B)) .
$$

Conversely, every general embedding of a $\Gamma$-covering of degree $n$ with 2-element base in $\mathbb{S B}(B)$ arises from an embedding of an étale extension in $B$.

Proof. The proof of the first (resp. second) part is similar to that of Proposition 2.6 (resp. 2.7).

Proposition 4.15 applies in particular in the situation of Theorem 4.8: for the rest of this subsection, suppose $A$ is a central simple $F$-algebra of degree $2 n$ 
with a quadratic pair $(\sigma, f)$. If $\left(L, \sigma_{L}\right)$ is an étale $F$-algebra with involution of dimension $2 n$ and

$$
\varepsilon:\left(L, \sigma_{L}\right) \hookrightarrow(A, \sigma, f)
$$

is an embedding of algebras with involution, then Theorem 4.8 yields an $F$-algebra embedding of the étale extension $C\left(L, \sigma_{L}\right) / \Delta(L)$ into $C(A, \sigma, f) / Z(A, \sigma, f)$. By Proposition 4.15 there is a general embedding

$$
C(\varepsilon)_{*}: \mathbf{X} C\left(L, \sigma_{L}\right) \hookrightarrow \mathbb{S B}(C(A, \sigma, f)) .
$$

On the other hand, the embedding $\varepsilon$ also yields by Proposition 3.8 an embedding of quadrics

$$
\varepsilon_{*}: \mathbf{Y}\left(L, \sigma_{L}\right) \hookrightarrow Q(\sigma, f) .
$$

To complete the picture, we now relate the Clifford $\Gamma$-set $C(Q(\sigma, f))$ to the Severi-Brauer double projective space $\mathbb{S B}(C(A, \sigma, f))$.

Recall from $[7, \S 8]$ that there is a Clifford bimodule $B(A, \sigma, f)$ over $C(A, \sigma, f)$, which is equipped with a canonical $F$-linear map $b: A \rightarrow B(A, \sigma, f)$, see [7, §9]. In the split case $A=\operatorname{End}_{F} V$, we have $B(A, \sigma, f)=V \otimes_{F} C_{1}(V, q)$. The bilinear polar form of $q$ yields an $F$-linear isomorphism $V \rightarrow V^{*}$ which allows us to identify

$$
V \otimes_{F} V=V \otimes_{F} V^{*}=\operatorname{End}_{F} V ;
$$

under this identification, the map $b$ carries $v \otimes w \in V \otimes_{F} V$ to $v \otimes w \in V \otimes_{F}$ $C_{1}(V, q)$.

Since $\operatorname{deg} A=2 n$, we have $\operatorname{dim}_{F} C(A, \sigma, f)=2^{2 n-1}$. For every $2 n$-dimensional isotropic right ideal $I \subset A_{s}$, let $\psi(I) \subset C\left(A_{s}, \sigma_{s}, f_{s}\right)$ denote the right annihilator of the $F$-vector space $\left(\sigma_{s}(I)\right)^{b} \subset B\left(A_{s}, \sigma_{s}, f_{s}\right)$, i.e.,

$$
\psi(I)=\left\{x \in C\left(A_{s}, \sigma_{s}, f_{s}\right) \mid\left(\sigma_{s}(I)\right)^{b} \cdot x=0 \text { for all } y \in I\right\} .
$$

Proposition 4.18. The map $\psi$ defines an embedding

$$
\psi:(\Delta(Q(\sigma, f)) \leftarrow C(Q(\sigma, f))) \hookrightarrow(\mathbf{X}(Z(A, \sigma, f)) \leftarrow \mathbb{S B}(C(A, \sigma, f))) .
$$

Proof. We first show $\operatorname{dim}_{F} \psi(I)=2^{n-1}$. Let $A_{s}=\operatorname{End}_{F_{s}} V$ for some $2 n$ dimensional $F_{s}$-vector space $V$. The quadratic pair $\left(\sigma_{s}, f_{s}\right)$ is adjoint to some hyperbolic quadratic form $q$ on $V$, and $I=\operatorname{Hom}_{F_{s}}(V, U)$ for some totally isotropic $n$-dimensional subspace $U \subset V$. We may therefore identify $V$ with $U \oplus U^{*}$, and $q$ with the quadratic form $q(u, \varphi)=\varphi(u)$ for $u \in U, \varphi \in U^{*}$. Then the full Clifford algebra of $q$ can be identified with the algebra $\operatorname{End}_{F_{s}}(\bigwedge U)$ of $F_{s}$-linear 
endomorphisms of the exterior algebra of $U$, in such a way that the canonical map

$$
V=U \oplus U^{*} \rightarrow C(V, q)=\operatorname{End}_{F_{s}}(\bigwedge U)
$$

maps $u \in U$ to the left exterior multiplication by $u$ and $\varphi \in U^{*}$ to its associated derivation $d_{\varphi}$, see $[7,(8.3)]$. Let

$$
\bigwedge_{0} U=\bigoplus_{i \text { even }} \bigwedge^{i} U \subset \wedge U \quad \text { and } \quad \bigwedge_{1} U=\bigoplus_{i \text { odd }} \bigwedge^{i} U \subset U .
$$

Then

$$
C\left(A_{s}, \sigma_{s}, f_{s}\right)=C_{0}(V, q)=\operatorname{End}_{F_{s}}\left(\bigwedge_{0} U\right) \times \operatorname{End}_{F_{s}}\left(\bigwedge_{1} U\right)
$$

and

$B\left(A_{s}, \sigma_{s}, f_{s}\right)=V \otimes_{F_{s}} C_{1}(V, q)=V \otimes_{F_{s}}\left(\operatorname{Hom}_{F_{s}}\left(\bigwedge_{0} U, \bigwedge_{1} U\right) \times \operatorname{Hom}_{F_{s}}\left(\bigwedge_{1} U, \bigwedge_{0} U\right)\right)$.

Under the identification $A_{s}=\operatorname{End}_{F_{s}} V=V \otimes_{F_{s}} V$ we have $I=U \otimes_{F_{s}} V$, hence

$$
\left(\sigma_{s}(I)\right)^{b}=V \otimes_{F_{s}} U \subset V \otimes_{F_{s}} C_{1}(V, q) .
$$

Therefore, $\psi(I)$ consists of the linear maps $f \in \operatorname{End}_{F_{s}}(\bigwedge U)$ such that $f\left(\bigwedge_{0} U\right) \subset$ $\bigwedge_{0} U, f\left(\bigwedge_{1} U\right) \subset \bigwedge_{1} U$, and

$$
u \wedge f(x)=0 \quad \text { for all } u \in U \text { and all } x \in \wedge U .
$$

The last condition implies $f(x) \in \bigwedge^{n} U$, hence

$$
\psi(I)= \begin{cases}\operatorname{Hom}_{F_{s}}\left(\bigwedge_{0} U, \bigwedge^{n} U\right) & \text { if } n \text { is even, } \\ \operatorname{Hom}_{F_{s}}\left(\bigwedge_{1} U, \bigwedge^{n} U\right) & \text { if } n \text { is odd }\end{cases}
$$

It follows that $\operatorname{dim}_{F_{s}} \psi(I)=\operatorname{dim}_{F_{s}} \bigwedge_{0} U=\operatorname{dim}_{F_{s}} \bigwedge_{1} U=2^{n-1}$, hence $\psi(I) \in$ $\mathbb{S B}(C(A, \sigma, f))$. Moreover, for every nonzero $\varphi \in U^{*}$ we have $d_{\varphi}\left(\bigwedge^{n} U\right) \neq\{0\}$, hence

$$
\{v \in V \mid v \cdot f=0 \text { in } C(V, q) \text { for all } f \in \psi(I)\}=U .
$$

It follows that

$$
I=\left\{y \in A \mid(\sigma(y))^{b} \cdot x=0 \text { for all } x \in \psi(I)\right\},
$$

hence the map $\psi$ is injective.

It is clear from the definition that the map $\psi$ is $\Gamma$-equivariant. To complete the proof, it remains to show that $\psi$ induces an isomorphism

$$
\bar{\psi}: \Delta(Q(\sigma, f)) \stackrel{\sim}{\longrightarrow} X(Z(A, \sigma, f)) .
$$


As above, we identify $A_{s}=\operatorname{End}_{F_{s}} V$ for some $2 n$-dimensional $F_{s}$-vector space $V$. Let $I, I^{\prime} \in C(Q(\sigma, f))$, so

$$
I=\operatorname{Hom}_{F_{s}}(V, U), \quad I^{\prime}=\operatorname{Hom}_{F_{s}}\left(V, U^{\prime}\right)
$$

for some totally isotropic $n$-dimensional subspaces $U, U^{\prime} \subset V$. By a theorem of Witt, there is an orthogonal transformation $g \in \mathrm{O}(V, q)$ such that $g(U)=U^{\prime}$. This transformation induces an automorphism $\hat{g}$ of $C_{0}(V, q)$ such that $\hat{g}(\psi(I))=$ $\psi\left(I^{\prime}\right)$. If $e$ (resp. $\left.e^{\prime}\right)$ is the primitive central idempotent of $C_{0}(V, q)$ such that $e \cdot \psi(I)=\psi(I)$ (resp. $\left.e^{\prime} \cdot \psi\left(I^{\prime}\right)=\psi\left(I^{\prime}\right)\right)$, we therefore have $\hat{g}(e)=e^{\prime}$. Now, we have $\operatorname{dim}\left(U \cap U^{\prime}\right) \equiv n \bmod 2$ if and only if $g \in \mathrm{O}^{+}(V, q)$ by [3, §§ II.6, II.10], and this condition holds if and only if the restriction of $\hat{g}$ to the center of $C_{0}(V, q)$ is the identity, by [7, (13.2)]. Therefore, $e=e^{\prime}$ if and only if the ideals $I$ and $I^{\prime}$ belong to the same class of $C(Q(\sigma, f))$. This completes the proof.

Corollary 4.19. If $(\sigma, f)$ is a hyperbolic quadratic pair on $A$, then the Clifford algebra $C(A, \sigma, f)$ decomposes into a direct product of simple F-algebras $C(A, \sigma, f) \simeq C_{1} \times C_{2}$, and one of the factors $C_{1}, C_{2}$ is split.

Proof. If $(\sigma, f)$ is hyperbolic, then its discriminant is trivial, hence $Z(A, \sigma, f) \simeq$ $F \times F$ and $C(A, \sigma, f)$ decomposes into a direct product of simple $F$-algebras as above. Moreover, there is a maximal isotropic linear variety in $Q(\sigma, f)$ that is defined over $F$, hence fixed under the action of $\Gamma$. Its image under $\psi$ is a point on $\mathbb{S B}(C(A, \sigma, f))=\mathcal{S B}\left(C_{1}\right) \sqcup \mathcal{S B}\left(C_{2}\right)$ fixed under the action of $\Gamma$. Its existence shows that $C_{1}$ or $C_{2}$ is split.

Remark 1. This corollary was first proved by D.C. Van Drooge and H.P. Allen, see also Tits [17, Proposition 8].

We may now relate the maps (4.16) and (4.17) associated to an embedding $\varepsilon$ of algebras with involution:

Proposition 4.20. The following diagram is commutative:

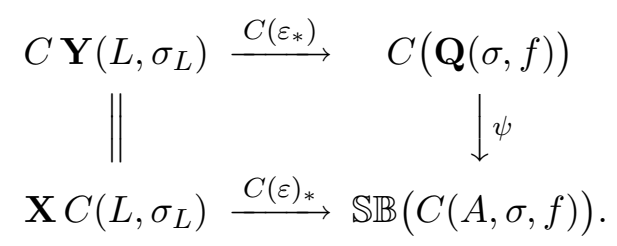


Proof. Extending scalars, we reduce to the case where $L$ is split, $A=\operatorname{End}_{F} L$, and $\varepsilon$ is the regular representation as in Example 3.6 (see Proposition 3.7). Let $\omega \in C \mathbf{Y}\left(L, \sigma_{L}\right)$, and let $e_{1}, \ldots, e_{n}, e_{1}^{\prime}, \ldots, e_{n}^{\prime}$ be the primitive idempotents of $L$, labelled in such a way that $\sigma\left(e_{i}\right)=e_{i}^{\prime}$ for $i \in \boldsymbol{n}$ and $\xi\left(e_{i}\right)=1, \xi\left(e_{i}^{\prime}\right)=0$ for $\xi \in \omega$. Under the identification $C \mathbf{Y}\left(L, \sigma_{L}\right)=X C\left(L, \sigma_{L}\right)$, the corresponding $F$-algebra homomorphism $\xi_{\omega}: C\left(L, \sigma_{L}\right) \rightarrow F$ satisfies

$$
\xi_{\omega}\left(c_{L}\left(e_{i}\right)\right)=1 \quad \text { and } \quad \xi_{\omega}\left(c_{L}\left(e_{i}^{\prime}\right)\right)=0 \quad \text { for all } i \in \boldsymbol{n} .
$$

In $C(A, \sigma, f)=C_{0}(L, t)$ we have $\varepsilon\left(e_{i}\right)=e_{i} e_{i}^{\prime}$ and $\varepsilon\left(e_{i}^{\prime}\right)=e_{i}^{\prime} e_{i}$ for $i \in \boldsymbol{n}$, hence

$$
C(\varepsilon)_{*}\left(\xi_{\omega}\right)=\left\{x \in C_{0}(L, t) \mid e_{i} e_{i}^{\prime} x=x \text { and } e_{i}^{\prime} e_{i} x=0 \text { for all } i \in \boldsymbol{n}\right\} .
$$

On the other hand, $C\left(\varepsilon_{*}\right)(\omega)=\operatorname{Hom}_{F}\left(L, \bigoplus_{i \in \boldsymbol{n}} e_{i} L\right)$, hence

$$
\psi\left(C\left(\varepsilon_{*}\right)(\omega)\right)=\left\{x \in C_{0}(L, t) \mid e_{i} \cdot x=0 \text { for } i \in \boldsymbol{n}\right\} .
$$

In $C(L, t)$, we have $e_{i}^{2}=0$ and $e_{i} e_{i}^{\prime}+e_{i}^{\prime} e_{i}=1$, hence $e_{i} e_{i}^{\prime} e_{i}=e_{i}$ for $i \in \boldsymbol{n}$. Therefore, if $e_{i}^{\prime} e_{i} x=0$, then $e_{i} x=0$. Conversely, if $e_{i} x=0$, then $e_{i}^{\prime} e_{i} x=0$ and

$$
x=\left(e_{i} e_{i}^{\prime}+e_{i}^{\prime} e_{i}\right) x=e_{i} e_{i}^{\prime} x .
$$

Therefore, $\psi\left(C\left(\varepsilon_{*}\right)(\omega)\right)=C(\varepsilon)_{*}(\omega)$.

\section{Dynkin diagrams and Severi-Brauer varieties}

The projective linear group $\mathrm{PGL}_{n}$ has Dynkin diagram of type $A_{n-1}$ and the group $\mathfrak{S}_{n}$ is its Weyl group. The special orthogonal group $\mathrm{PGO}_{2 n}^{+}$, with Weyl group $\mathfrak{S}_{2}^{n-1} \rtimes \mathfrak{S}_{n}$, has Dynkin diagram of type $D_{n}$. Accordingly we call groupoids occurring in Proposition 2.2 and (2.3) groupoids of type $A_{n-1}$, and groupoids occurring in (3.4) and Proposition 3.1 groupoids of type $D_{n}$. Many properties of these groupoids are related to properties of the corresponding Dynkin diagrams. After introducing oriented quadrics and étale algebras, we briefly consider two examples: the equivalence $A_{3}=D_{3}$ and triality.

Special quadrics. A special or oriented $\Gamma$-quadric is a pair $(Q, \partial)$, where $Q$ is $\Gamma$-quadric (or a thin $\Gamma$-quadric) and $\partial$ is a fixed isomorphism of $\Gamma$-sets $\mathbf{2} \simeq \Delta(Q)$. In particular the $\Gamma$-action on $\Delta(Q)$ is trivial. There are two possible choices for $\partial$. A choice is an orientation of $Q$. If $Q$ is oriented we may use the given isomorphism $\partial: \mathbf{2} \simeq \Delta(Q)$ to decompose the Clifford $\Gamma$-set:

$$
C\left(Z / Z_{0}\right)=C_{1}\left(Z / Z_{0}, \partial\right) \sqcup C_{2}\left(Z / Z_{0}, \partial\right)
$$


where

$$
C_{i}\left(Z / Z_{0}, \partial\right)=\left\{\omega \in C\left(Z / Z_{0}\right) \mid \omega^{\delta \partial}=i\right\} \quad \text { for } i=1,2 .
$$

Similarly, we define oriented extensions of étale $F$-algebras as pairs $\left(L / L_{0}, \partial\right)$ where $L / L_{0}$ is an extension of étale $F$-algebras and $\partial$ is an orientation of $L$, i.e., a fixed isomorphism $\partial: \Delta(L) \stackrel{\sim}{\longrightarrow} F \times F$. We also have oriented central simple algebras with quadratic pairs, defined as 4-tuples $(A, \sigma, f, \partial)$, where $\partial$ is a fixed isomorphism $\partial: Z(A, \sigma, f) \stackrel{\sim}{\longrightarrow} F \times F$. In the corresponding decomposition

$$
C(A, \sigma, f, \partial)=C_{1}(A, \sigma, f, \partial) \times C_{2}(A, \sigma, f, \partial),
$$

$C_{1}(A, \sigma, f, \partial)$ and $C_{2}(A, \sigma, f, \partial)$ are central simple algebras over $F$. The classes of morphisms considered in the previous sections can be restricted to preserve the orientations. For example an embedding of oriented quadrics

$$
\varepsilon:\left(Y, \sigma, \partial_{Y}\right) \hookrightarrow\left(Q, \partial_{Q}\right)
$$

is an embedding $\varepsilon: Y \hookrightarrow Q$ such that $\Delta(\varepsilon) \circ \partial_{Q}=\partial_{Y}$. The diagram (4.21) restricts to a commutative diagram

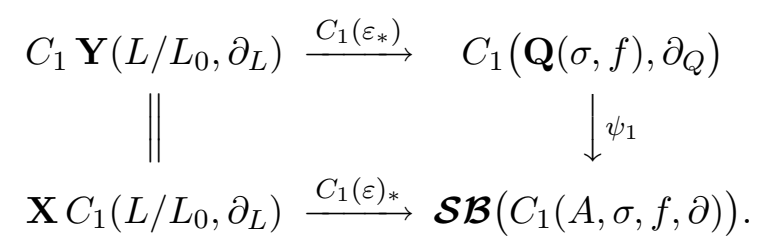

As in 2.2, 2.3, 3.4 and 3.1, we have anti-equivalences of groupoids

$$
\mathrm{OQCSA}_{F}^{2 n} \equiv \operatorname{OQuad}_{\Gamma}^{2(n-1)} \text { and } \mathrm{OQÉ}_{\mathrm{tex}}^{n} \equiv \mathrm{OQCov}_{\Gamma}^{n} \text {, }
$$

using the following notations:

(1) OQCSA ${ }_{F}^{2 n}$ for the groupoid of oriented central simple algebras over $F$ of degree $2 n$ with quadratic pairs,

(2) $\mathrm{OQuad}_{\Gamma}^{2(n-1)}$ for the groupoid of oriented $\Gamma$-quadrics of dimension $2(n-1)$ over $F$,

(3) OQÉtex ${ }_{F}^{n}$ for the groupoid of oriented quadratic étale extensions $L / L_{0}$ of $F$-algebras with $\operatorname{dim}_{F} L_{0}=n$.

(4) $\mathrm{OQCov}_{\Gamma}^{n}$ for the groupoid of oriented thin $\Gamma$-quadrics of dimension $2(n-1)$ over $F$, i.e., double $\Gamma$-coverings $Y / Y_{0}$ with $\left|Y_{0}\right|=n$.

Isomorphism classes are in bijection with $H^{1}\left(\Gamma, \mathrm{PGO}_{2 n}^{+}\right)$, resp. $H^{1}\left(\Gamma, \mathfrak{S}_{2}^{n-1} \rtimes \mathfrak{S}_{n}\right)$. 
The exceptional isomorphism $A_{3}=D_{3}$. The fact that the Dynkin diagrams $A_{3}$ and $D_{3}$ are identical can be interpreted at the level of the corresponding groupoids. For any 4 -element $\Gamma$-set $X$, the $\Gamma$-set $G r_{1}(X)$ of 2-element subsets of $X$ is equipped with a canonical involution $\sigma$, which maps each 2-element subset of $X$ to its complement. Thus, it defines a functor $\mathrm{Set}_{\Gamma}^{4} \rightarrow \mathrm{QCov}_{\Gamma}^{3}$, which we also designate by $G r_{1}$. The sections $\left\{S_{1}, S_{2}, S_{3}\right\} \in C\left(G r_{1}(X), \sigma\right)$ (where $S_{1}, S_{2}, S_{3}$ are 2-element subsets of $X$ such that $\left.\left\{\sigma\left(S_{1}\right), \sigma\left(S_{2}\right), \sigma\left(S_{3}\right)\right\} \cap\left\{S_{1}, S_{2}, S_{3}\right\}=\emptyset\right)$ naturally fall into two classes, depending on whether $S_{1} \cap S_{2} \cap S_{3}$ is empty or is a 1-element subset of $X$. Therefore, there is a natural orientation on $G r_{1}(X)$, and we may view $G r_{1}$ as a functor $\operatorname{Set}_{\Gamma}^{4} \rightarrow \mathrm{OQCov}_{\Gamma}^{3}$.

The analogue of $G r_{1}$ for étale algebras under the Grothendieck correspondence is an "exterior algebra" functor $\lambda^{2}:$ Ét $_{F}^{4} \rightarrow \mathrm{OQÉtex}_{F}^{3}$ studied in [8]. There is also an "exterior algebra" functor $\lambda^{2}: \mathrm{CSA}_{F}^{4} \rightarrow \mathrm{OQCSA}_{F}^{6}$ discussed in $[7, \S 15 \mathrm{D}]$.

Proposition 5.1. The functors $G r_{1}$ (resp. $\left.\lambda^{2}\right)$ and $C_{1}$ induce equivalences:

$$
\begin{array}{cc}
\operatorname{Set}_{\Gamma}^{4} \underset{C_{1}}{\stackrel{G r_{1}}{\rightleftarrows}} \text { OQCov }_{\Gamma}^{3}, & \text { Ét }_{F}^{4} \underset{C_{1}}{\stackrel{\lambda^{2}}{\rightleftarrows}} \text { OQÉtex }_{F}^{3}, \\
\operatorname{Proj}_{\Gamma}^{3} \underset{C_{1}}{\stackrel{G r_{1}}{\rightleftarrows}} \text { OQuad }_{\Gamma}^{4}, & \text { CSA }_{F}^{4} \underset{C_{1}}{\stackrel{\lambda^{2}}{\rightleftarrows}} \text { OQCSA }_{\Gamma}^{6} .
\end{array}
$$

Moreover these equivalences are compatible with general embeddings.

Proof. The (equivalent) first two cases are discussed in [8], the third is equivalent to the last and the last follows from $[7,(15.32)]$.

Remark 5.2. The equivalence between groupoids of thin objects of type $A_{3}$ and of type $D_{3}$ is induced at the cohomological level by the well-known isomorphism $\mathfrak{S}_{4} \stackrel{\sim}{\longrightarrow} \mathfrak{S}_{2}^{2} \rtimes \mathfrak{S}_{3}$. Classically, for any separable field extension $E$ of degree 4 , the subalgebra of $\lambda^{2} E$ fixed under the involution is the cubic resolvent (see [8]). On the geometric side, the quadric $G r_{1}(P)$ can be viewed as a 4-dimensional quadric parametrised by a 3-dimensional projective space. This quadric is known as Klein's quadric.

Triality. All Dynkin diagrams but one admit at most automorphisms of order two, which are related to duality in algebra and geometry. The Dynkin diagram of $D_{4}$ : 


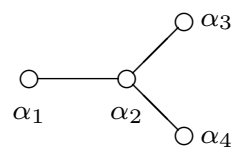

is special, in the sense that it admits automorphisms of order 3. Algebraic and geometric objects related to $D_{4}$ are of particular interest as they also usually admit exceptional automorphisms of order 3, which are called trialitarian. This is the case for the four groupoids OQCov ${ }_{\Gamma}^{4}, \mathrm{OQÉ}_{\text {tex }}^{4}$, OQuad ${ }_{\Gamma}^{6}$ and OQCSA ${ }_{F}^{8}$ of type $D_{4}$. For $\left(E, \partial_{E}\right)$ in OQÉtex ${ }_{F}^{4}$ or in OQCSA ${ }_{F}^{8}$, the objects $C_{1}\left(E, \partial_{E}\right)$ and $C_{2}\left(E, \partial_{E}\right)$ belong to QÉtex ${ }_{F}^{4}$, resp. to QCSA ${ }_{F}^{8}$ (see [9], resp. [7, §35]). Corresponding results hold for objects in $\mathrm{OQCov}_{\Gamma}^{4}$ or $\mathrm{OQuad}_{\Gamma}^{6}$ since these groupoids of quadrics are anti-equivalent to the corresponding groupoids of algebras. Isomorphism classes of these groupoids are in bijection with $H^{1}\left(\Gamma, \mathrm{PGO}_{4}^{+}\right)$or $H^{1}\left(\Gamma, \mathfrak{S}_{2}^{3} \rtimes \mathfrak{S}_{4}\right)$. The groups $\mathrm{PGO}_{8}^{+}$and $\mathfrak{S}_{2}^{3} \rtimes \mathfrak{S}_{4}$ admit up to inner automorphisms one outer action of the cyclic group of order 3 (this is well known for $\mathrm{PGO}_{8}^{+}$and is in [5] for $\mathfrak{S}_{2}^{3} \rtimes \mathfrak{S}_{4}$ ). Thus the cyclic group of order 3 acts on the corresponding sets of isomorphism classes. In fact these automorphisms of order 3 are induced by automorphisms that can already be defined at the level of the groupoids. Let $\mathrm{D}_{4}$ be any of the four groupoids above, let $\widetilde{\mathrm{D}}_{4}$ be the corresponding groupoid of unoriented objects, and let $\mathcal{F}:\left(E, \partial_{E}\right) \mapsto E$ be the forgetful functor $\mathrm{D}_{4} \rightarrow \widetilde{\mathrm{D}}_{4}$.

Theorem 5.3. The functors $C_{1}, C_{2}$ factor through the forgetful functor, i.e., there are functors

$$
C_{1}^{+}, C_{2}^{+}: \mathrm{D}_{4} \rightarrow \mathrm{D}_{4}
$$

such that $\mathcal{F} \circ C_{i}^{+}=C^{i}$ for $i=1,2$. These functors satisfy natural equivalences:

$$
\left(C_{1}^{+}\right)^{3}=\mathrm{Id}, \quad\left(C_{1}^{+}\right)^{2}=C_{2}^{+}
$$

and induce the trialitarian action on the sets of isomorphism classes.

Proof. The claim for thin objects is in [9] (under the hypothesis that $\operatorname{char}(F) \neq 2$ ) and the classical case (for algebras) is in [7, §35].

Observe that triality is compatible with general embeddings. Triality for thin objects is extensively discussed in [9]. A very nice presentation of classical (geometric and algebraic) triality is in [20]. 


\section{REFERENCES}

[1] R. Brusamarello, P. Chuard-Koulmann, and J. Morales. Orthogonal groups containing a given maximal torus. J. Algebra, 266:87-101, 2003.

[2] P. Deligne. Séminaire de géométrie algébrique du Bois-Marie, SGA 4 1/2, Cohomologie étale, avec la collaboration de J. F. Boutot, A. Grothendieck, L. Illusie et J. L. Verdier, volume 569 of Lecture Notes in Mathematics. Springer-Verlag, 1977.

[3] J. Dieudonné. La géométrie des groupes classiques. Seconde édition, revue et corrigée. Springer-Verlag, Berlin, 1963.

[4] D. Ferrand. Un foncteur norme. Bull. Soc. math. France, 126:1-49, 1998.

[5] W. N. Franzsen and R. B. Howlett. Automorphisms of nearly finite Coxeter groups. Adv. Geom., 3(3):301-338, 2003.

[6] J. Jahnel. The Brauer-Severi variety associated with a central simple algebra: A survey. Preprint 52, Linear Algebraic Groups and Related Structures Preprint Server, http://www.mathematik.uni- bielefeld.de/LAG/man/052.html, 2000.

[7] M-A. Knus, A. A. Merkurjev, M. Rost, and J-P. Tignol. The Book of Involutions. Number 44 in American Mathematical Society Colloquium Publications. American Mathematical Society, Providence, R.I., 1998. With a preface in French by J. Tits.

[8] M-A. Knus and J-P. Tignol. Quartic exercises. Inter. J. Math. Math. Sci., 2003:4263-4323, 2003.

[9] M-A. Knus and J-P. Tignol. Triality and étale algebras. In: J.-L. Colliot-Thélène, S. Garibaldi, R. Sujatha, and V. Suresh, editors. Quadratic forms, linear algebraic groups, and cohomology, Developments in Mathematics. Springer, 2010.

[10] M-A. Knus and J-P. Tignol. Severi-Brauer varieties over the field of characteristic one. In preparation, 2010.

[11] S. Mac Lane. Categories for the Working Mathematician, volume 5 of Graduate Texts in Mathematics. Springer-Verlag, 2nd ed., 1998.

[12] A. S. Merkurjev. Closed points of Severi-Brauer varieties. Vestnik S.-Peterburg. Univ. Mat. Mekh. Astronom., (vyp. 2):51-53, 124, 1993.

[13] G. Prasad and A. S. Rapinchuk. Local-global principles for embedding of fields with involution into simple algebras with involution. Commentarii mathematici Helvetici 85:485-534, 2010 .

[14] D. J Saltman. Triality, cocycles, crossed products, involutions, Clifford algebras and invariants, 88 p. Austin.

[15] J-P. Serre. Corps locaux. Number VIII in Publications de l'Institut de Mathématique de l'Université de Nancago. Hermann, Paris, 1968.

[16] J. Tits. Sur les analogues algébriques des groupes semi-simples complexes. In Colloque d'algèbre supérieure, tenu à Bruxelles du 19 au 22 décembre 1956, pages 261-289. Centre Belge de Recherches Mathématiques, Établissements Ceuterick, Louvain; Librairie Gauthier-Villars, Paris, 1957.

[17] J. Tits. Formes quadratiques, groupes orthogonaux et algèbres de Clifford. Invent. Math., 5:19-41, 1968. 
[18] J. Tits. Buildings of Spherical Type and Finite BN-Pairs, volume 386 of Lecture Notes in Mathematics. Springer-Verlag, 1974.

[19] J. Tits. Sur les produits tensoriels de deux algèbres de quaternions. Bull. Soc. Math. Belg. Sér. B 45:329-331, 1993.

[20] F. van der Blij and T.A. Springer. Octaves and triality. Nieuw Arch. Wiskd., III. Ser., 8:158-169, 1960.

Max-Albert Knus

Department Mathematik

ETH Zentrum, CH-8092 Zürich

Switzerland

Email: knus@math.ethz.ch

Jean-Pierre Tignol

Institut de Mathématique Pure et Appliquée

Université catholique de Louvain, B-1348 Louvain-la-Neuve

Belgium

E-mail: jean-pierre.tignol@uclouvain.be 